\title{
Middle Convolution and Heun's Equation ${ }^{\star}$
}

\author{
Kouichi TAKEMURA
}

Department of Mathematical Sciences, Yokohama City University, 22-2 Seto, Kanazawa-ku, Yokohama 236-0027, Japan

E-mail: takemura@yokohama-cu.ac.jp

Received November 26, 2008, in final form March 25, 2009; Published online April 03, 2009 doi:10.3842/SIGMA.2009.040

\begin{abstract}
Heun's equation naturally appears as special cases of Fuchsian system of differential equations of rank two with four singularities by introducing the space of initial conditions of the sixth Painlevé equation. Middle convolutions of the Fuchsian system are related with an integral transformation of Heun's equation.
\end{abstract}

Key words: Heun's equation; the space of initial conditions; the sixth Painlevé equation; middle convolution

2000 Mathematics Subject Classification: 34M35; 33E10; 34M55

\section{Introduction}

Heun's equation is a standard form of a second-order Fuchsian differential equation with four singularities, and it is given by

$$
\frac{d^{2} y}{d z^{2}}+\left(\frac{\gamma}{z}+\frac{\delta}{z-1}+\frac{\epsilon}{z-t}\right) \frac{d y}{d z}+\frac{\alpha \beta z-q}{z(z-1)(z-t)} y=0
$$

with the condition

$$
\gamma+\delta+\epsilon=\alpha+\beta+1
$$

The parameter $q$ is called an accessory parameter. Although the local monodromy (local exponent) is independent of $q$, the global monodromy (e.g. the monodromy on the cycle enclosing two singularities) depends on $q$. Some properties of Heun's equation are written in the books [21, 23], but an important feature related with the theory of finite-gap potential for the case $\gamma, \delta, \epsilon, \alpha-\beta \in$ $\mathbb{Z}+\frac{1}{2}$ (see $[6,24,25,26,27,28,29,31]$ etc.), which leads to an algorithm to calculate the global monodromy explicitly for all $q$, is not written in these books.

The sixth Painlevé equation is a non-linear ordinary differential equation written as

$$
\begin{aligned}
\frac{d^{2} \lambda}{d t^{2}}= & \frac{1}{2}\left(\frac{1}{\lambda}+\frac{1}{\lambda-1}+\frac{1}{\lambda-t}\right)\left(\frac{d \lambda}{d t}\right)^{2}-\left(\frac{1}{t}+\frac{1}{t-1}+\frac{1}{\lambda-t}\right) \frac{d \lambda}{d t} \\
& +\frac{\lambda(\lambda-1)(\lambda-t)}{t^{2}(t-1)^{2}}\left\{\frac{\left(1-\theta_{\infty}\right)^{2}}{2}-\frac{\theta_{0}^{2}}{2} \frac{t}{\lambda^{2}}+\frac{\theta_{1}^{2}}{2} \frac{(t-1)}{(\lambda-1)^{2}}+\frac{\left(1-\theta_{t}^{2}\right)}{2} \frac{t(t-1)}{(\lambda-t)^{2}}\right\} .
\end{aligned}
$$

A remarkable property of this differential equation is that the solutions do not have movable singularities other than poles. It is known that the sixth Painlevé equation is obtained by monodromy preserving deformation of Fuchsian system of differential equations,

$$
\frac{d}{d z}\left(\begin{array}{l}
y_{1} \\
y_{2}
\end{array}\right)=\left(\frac{A_{0}}{z}+\frac{A_{1}}{z-1}+\frac{A_{t}}{z-t}\right)\left(\begin{array}{l}
y_{1} \\
y_{2}
\end{array}\right), \quad A_{0}, A_{1}, A_{t} \in \mathbb{C}^{2 \times 2} .
$$

* This paper is a contribution to the Proceedings of the Workshop "Elliptic Integrable Systems, Isomonodromy Problems, and Hypergeometric Functions" (July 21-25, 2008, MPIM, Bonn, Germany). The full collection is available at http://www.emis.de/journals/SIGMA/Elliptic-Integrable-Systems.html 
See Section 2 for expressions of the elements of the matrices $A_{0}, A_{1}, A_{t}$. By eliminating $y_{2}$ we have second-order differential equation for $y_{1}$, which have an additional apparent singularity $z=\lambda$ other than $\{0,1, t, \infty\}$ for generic cases, and the point $\lambda$ corresponds to the variable of the sixth Painlevé equation. For details of monodromy preserving deformation, see [10]. In this paper we investigate the condition that the second-order differential equation for $y_{1}$ is written as Heun's equation. To get a preferable answer, we introduce the space of initial conditions for the sixth Painlevé equation which was discovered by Okamoto [18] to construct a suitable defining variety for the set of solutions to the (sixth) Painlevé equation.

For Fuchsian systems of differential equations and local systems on a punctured Riemann sphere, Dettweiler and Reiter [2,3] gave an algebraic analogue of Katz' middle convolution functor [12]. Filipuk [5] applied them for the Fuchsian systems with four singularities, obtained an explicit relationship with the symmetry of the sixth Painlevé equation, and the author [30] calculated the corresponding integral transformation for the Fuchsian systems with four singularities. The middle convolution is labeled by a parameter $\nu$, and we have two values which leads to non-trivial transformation on $2 \times 2$ Fuchsian system with four singularities (see Section 4 ). In this paper we consider the middle convolution which is a different value of the parameter $\nu$ from the one discussed in $[5,30]$. We will also study the relationship between middle convolution and Heun's equation. For special cases, the integral transformation raised by the middle convolution turns out to be a transformation on Heun's equation, and we investigate these cases. Note that the description by the space of initial conditions for the sixth Painlevé equation is favorable. The integral transformation of Heun's equation is applied for the study of novel solutions, which we will discuss in a separated publication. If the parameter of the middle convolution is a negative integer, then the integral transformation changes to a successive differential, and a transformation defined by a differential operator on Heun's equation was found in [29] as a generalized Darboux transformation (Crum-Darboux transformation). Hence the integral transformation on Heun's equation can be regarded as a generalization of the generalized Darboux transformation, which is related with the conjectual duality by Khare and Sukhatme [15].

Special functions of the isomonodromy type including special solutions to the sixth Painlevé equation have been studied actively and they are related with various objects in mathematics and physics $[16,32]$. On the other hand, special functions of Fuchsian type including special solutions to Heun's equation are also interesting objects which are related with general relativity and so on. This paper is devoted to an attempt to clarify both sides of viewpoints.

This paper is organized as follows: In Section 2, we fix notations for the Fuchsian system with four singularities. In Section 3, we define the space of initial conditions for the sixth Painlevé equation and observe that Heun's equation is obtained from the Fuchsian equation by restricting to certain lines in the space of initial conditions. In Section 4, we review results on the middle convolution and construct integral transformations. In Section 5, we investigating relationship among the middle convolution, integral transformations of Heun's equation and the space of initial conditions. In Section 6, we consider the case that the parameter on the middle convolution is integer. In the appendix, we describe topics which was put off in the text.

\section{Fuchsian system of rank two with four singularities}

We consider a system of ordinary differential equations,

$$
\frac{d Y}{d z}=A(z) Y, \quad A(z)=\frac{A_{0}}{z}+\frac{A_{1}}{z-1}+\frac{A_{t}}{z-t}=\left(\begin{array}{cc}
a_{11}(z) & a_{12}(z) \\
a_{21}(z) & a_{22}(z)
\end{array}\right), \quad Y=\left(\begin{array}{l}
y_{1} \\
y_{2}
\end{array}\right)
$$

where $t \neq 0,1, A_{0}, A_{1}, A_{t}$ are $2 \times 2$ matrix with constant elements. Then equation (2.1) is Fuchsian, i.e., any singularities on the Riemann sphere $\mathbb{C} \cup\{\infty\}$ are regular, and it may 
have regular singularities at $z=0,1, t, \infty$ on the Riemann sphere $\mathbb{C} \cup\{\infty\}$. Exponents of equation (2.1) at $z=0$ (resp. $z=1, z=t, z=\infty$ ) are described by eigenvalues of the matrix $A_{0}$ (resp. $\left.A_{1}, A_{t},-\left(A_{0}+A_{1}+A_{t}\right)\right)$. By the transformation $Y \rightarrow z^{n_{0}}(z-1)^{n_{1}}(z-t)^{n_{t}} Y$, the system of differential equations (2.1) is replaced as $A(z) \rightarrow A(z)+\left(n_{0} / z+n_{1} /(z-1)+n_{2} /(z-t)\right) I$ ( $I$ : unit matrix), and we can transform equation (2.1) to the one where one of the eigenvalues of $A_{i}$ is zero for $i \in\{0,1, t\}$ by putting $-n_{i}$ to be one of the eigenvalues of the original $A_{i}$. If the exponents at $z=\infty$ are distinct, then we can normalize the matrix $-\left(A_{0}+A_{1}+A_{t}\right)$ to be diagonal by a suitable gauge transformation $Y \rightarrow G Y, A(z) \rightarrow G A(z) G^{-1}$. In this paper we assume that one of the eigenvalues of $A_{i}$ is zero for $i=0,1, t$ and the matrix $-\left(A_{0}+A_{1}+A_{t}\right)$ is diagonal, and we set

$$
A_{\infty}=-\left(A_{0}+A_{1}+A_{t}\right)=\left(\begin{array}{cc}
\kappa_{1} & 0 \\
0 & \kappa_{2}
\end{array}\right)
$$

By eliminating $y_{2}$ in equation (2.1), we have a second-order linear differential equation,

$$
\begin{aligned}
& \frac{d^{2} y_{1}}{d z^{2}}+p_{1}(z) \frac{d y_{1}}{d z}+p_{2}(z) y_{1}=0, \quad p_{1}(z)=-a_{11}(z)-a_{22}(z)-\frac{\frac{d}{d z} a_{12}(z)}{a_{12}(z)}, \\
& p_{2}(z)=a_{11}(z) a_{22}(z)-a_{12}(z) a_{21}(z)-\frac{d}{d z} a_{11}(z)+\frac{a_{11}(z) \frac{d}{d z} a_{12}(z)}{a_{12}(z)} .
\end{aligned}
$$

Set

$$
A_{i}=\left(\begin{array}{ll}
a_{11}^{(i)} & a_{12}^{(i)} \\
a_{21}^{(i)} & a_{22}^{(i)}
\end{array}\right), \quad(i=0,1, t)
$$

It follows from equation $(2.2)$ that $a_{12}^{(0)}+a_{12}^{(1)}+a_{12}^{(t)}=0, a_{21}^{(0)}+a_{21}^{(1)}+a_{21}^{(t)}=0$. Hence $a_{12}(z)$ and $a_{21}(z)$ are expressed as

$$
a_{12}(z)=\frac{k_{1} z+k_{2}}{z(z-1)(z-t)}, \quad a_{21}(z)=\frac{\tilde{k}_{1} z+\tilde{k}_{2}}{z(z-1)(z-t)},
$$

and we have

$$
\begin{array}{lll}
a_{12}^{(0)}+a_{12}^{(1)}+a_{12}^{(t)}=0, & (t+1) a_{12}^{(0)}+t a_{12}^{(1)}+a_{12}^{(t)}=-k_{1}, & t a_{12}^{(0)}=k_{2}, \\
a_{21}^{(0)}+a_{21}^{(1)}+a_{21}^{(t)}=0, & (t+1) a_{21}^{(0)}+t a_{21}^{(1)}+a_{21}^{(t)}=-\tilde{k}_{1}, & t a_{21}^{(0)}=\tilde{k}_{2} .
\end{array}
$$

If $k_{1}=k_{2}=0$, then $y_{1}$ satisfies a first-order linear differential equation, and it is integrated easily. Hence we assume that $\left(k_{1}, k_{2}\right) \neq(0,0)$. Then it is shown that two of $a_{12}^{(0)}, a_{12}^{(1)}, a_{12}^{(t)}$, $(t+1) a_{12}^{(0)}+t a_{12}^{(1)}+a_{12}^{(t)}$ cannot be zero. We set $\lambda=-k_{2} / k_{1}\left(k_{1} \neq 0\right)$ and $\lambda=\infty\left(k_{1}=0\right)$. The condition that none of $a_{12}^{(0)}, a_{12}^{(1)}, a_{12}^{(t)}$ nor $(t+1) a_{12}^{(0)}+t a_{12}^{(1)}+a_{12}^{(t)}$ is zero is equivalent to that $\lambda \neq 0,1, t, \infty$, and the condition $a_{12}^{(0)}=0\left(\right.$ resp. $\left.a_{12}^{(1)}=0, a_{12}^{(t)}=0,(t+1) a_{12}^{(0)}+t a_{12}^{(1)}+a_{12}^{(t)}=0\right)$ is equivalent to $\lambda=0$ (resp. $\lambda=1, \lambda=t, \lambda=\infty$ ).

We consider the case $\lambda \neq 0,1, t, \infty$, i.e., the case $a_{12}^{(0)} \neq 0, a_{12}^{(1)} \neq 0, a_{12}^{(t)} \neq 0,(t+1) a_{12}^{(0)}+$ $t a_{12}^{(1)}+a_{12}^{(t)} \neq 0$. Let $\theta_{0}$ (resp. $\theta_{1}, \theta_{t}$ ) and 0 be the eigenvalues of $A_{0}$ (resp. $A_{1}, A_{t}$ ). Then we can set $A_{0}, A_{1}, A_{t}$ as

$$
\begin{aligned}
A_{0} & =\left(\begin{array}{ll}
u_{0}+\theta_{0} & -w_{0} \\
u_{0}\left(u_{0}+\theta_{0}\right) / w_{0} & -u_{0}
\end{array}\right), \quad A_{1}=\left(\begin{array}{ll}
u_{1}+\theta_{1} & -w_{1} \\
u_{1}\left(u_{1}+\theta_{1}\right) / w_{1} & -u_{1}
\end{array}\right), \\
A_{t} & =\left(\begin{array}{ll}
u_{t}+\theta_{t} & -w_{t} \\
u_{t}\left(u_{t}+\theta_{t}\right) / w_{t} & -u_{t}
\end{array}\right),
\end{aligned}
$$


by introducing variables $u_{0}, w_{0}, u_{1}, w_{1}, u_{t}, w_{t}$. By taking trace of equation (2.2), we have the relation $\theta_{0}+\theta_{1}+\theta_{t}+\kappa_{1}+\kappa_{2}=0$. We set $\theta_{\infty}=\kappa_{1}-\kappa_{2}$, then we have $\kappa_{1}=\left(\theta_{\infty}-\theta_{0}-\theta_{1}-\theta_{t}\right) / 2$, $\kappa_{2}=-\left(\theta_{\infty}+\theta_{0}+\theta_{1}+\theta_{t}\right) / 2$.

We determine $u_{0}, u_{1}, u_{t}, w_{0}, w_{1}, w_{t}$ so as to satisfy equation (2.2) and the following relations:

$$
\begin{aligned}
& a_{12}(z)=-\frac{w_{0}}{z}-\frac{w_{1}}{z-1}-\frac{w_{t}}{z-t}=\frac{k(z-\lambda)}{z(z-1)(z-t)}, \\
& a_{11}(\lambda)=\frac{u_{0}+\theta_{0}}{\lambda}+\frac{u_{1}+\theta_{1}}{\lambda-1}+\frac{u_{t}+\theta_{t}}{\lambda-t}=\mu,
\end{aligned}
$$

(see [11]). Namely, we solve the following equations for $u_{0}, u_{1}, u_{t}, w_{0}, w_{1}, w_{t}$ :

$$
\begin{aligned}
& -w_{0}-w_{1}-w_{t}=0, \quad w_{0}(t+1)+w_{1} t+w_{t}=k, \quad-w_{0} t=-k \lambda, \\
& u_{0}\left(u_{0}+\theta_{0}\right) / w_{0}+u_{1}\left(u_{1}+\theta_{1}\right) / w_{1}+u_{t}\left(u_{t}+\theta_{t}\right) / w_{t}=0, \\
& u_{0}+\theta_{0}+u_{1}+\theta_{1}+u_{t}+\theta_{t}=-\kappa_{1}, \quad-u_{0}-u_{1}-u_{t}=-\kappa_{2}, \\
& \left(u_{0}+\theta_{0}\right) / \lambda+\left(u_{1}+\theta_{1}\right) /(\lambda-1)+\left(u_{t}+\theta_{t}\right) /(\lambda-t)=\mu .
\end{aligned}
$$

The linear equations for $w_{0}, w_{1}, w_{t}$ are solved as

$$
w_{0}=\frac{k \lambda}{t}, \quad w_{1}=-\frac{k(\lambda-1)}{t-1}, \quad w_{t}=\frac{k(\lambda-t)}{t(t-1)} .
$$

By the equations which are linear in $u_{0}, u_{1}$ and $u_{t}$, we can express $u_{1}+\theta_{1}$ and $u_{t}+\theta_{t}$ as linear functions in $u_{0}$. We substitute $u_{1}+\theta_{1}$ and $u_{t}+\theta_{t}$ into a quadratic equation in $u_{0}, u_{1}$ and $u_{t}$. Then the coefficient of $u_{0}^{2}$ disappears, and $u_{0}, u_{1}, u_{t}$ are solved as

$$
\begin{aligned}
u_{0}= & -\theta_{0}+\frac{\lambda}{t \theta_{\infty}}\left[\lambda(\lambda-1)(\lambda-t) \mu^{2}+\left\{2 \kappa_{1}(\lambda-1)(\lambda-t)-\theta_{1}(\lambda-t)\right.\right. \\
& \left.\left.-t \theta_{t}(\lambda-1)\right\} \mu+\kappa_{1}\left\{\kappa_{1}(\lambda-t-1)-\theta_{1}-t \theta_{t}\right\}\right], \\
u_{1}= & -\theta_{1}-\frac{\lambda-1}{(t-1) \theta_{\infty}}\left[\lambda(\lambda-1)(\lambda-t) \mu^{2}+\left\{2 \kappa_{1}(\lambda-1)(\lambda-t)+\left(\theta_{\infty}-\theta_{1}\right)(\lambda-t)\right.\right. \\
& \left.\left.-t \theta_{t}(\lambda-1)\right\} \mu+\kappa_{1}\left\{\kappa_{1}(\lambda-t+1)+\theta_{0}-(t-1) \theta_{t}\right\}\right], \\
u_{t}= & -\theta_{t}+\frac{\lambda-t}{t(t-1) \theta_{\infty}}\left[\lambda(\lambda-1)(\lambda-t) \mu^{2}+\left\{2 \kappa_{1}(\lambda-1)(\lambda-t)-\theta_{1}(\lambda-t)\right.\right. \\
& \left.\left.+t\left(\theta_{\infty}-\theta_{t}\right)(\lambda-1)\right\} \mu+\kappa_{1}\left\{\kappa_{1}(\lambda-t+1)+\theta_{0}+(t-1)\left(\theta_{\infty}-\theta_{t}\right)\right\}\right] .
\end{aligned}
$$

We denote the Fuchsian system of differential equations

$$
\frac{d Y}{d z}=\left(\frac{A_{0}}{z}+\frac{A_{1}}{z-1}+\frac{A_{t}}{z-t}\right) Y, \quad Y=\left(\begin{array}{c}
y_{1} \\
y_{2}
\end{array}\right)
$$

with equations $(2.5),(2.7),(2.8)$ by $D_{Y}\left(\theta_{0}, \theta_{1}, \theta_{t}, \theta_{\infty} ; \lambda, \mu ; k\right)$. Then the second-order differential equation (2.3) is written as

$$
\begin{aligned}
\frac{d^{2} y_{1}}{d z^{2}} & +\left(\frac{1-\theta_{0}}{z}+\frac{1-\theta_{1}}{z-1}+\frac{1-\theta_{t}}{z-t}-\frac{1}{z-\lambda}\right) \frac{d y_{1}}{d z} \\
& +\left(\frac{\kappa_{1}\left(\kappa_{2}+1\right)}{z(z-1)}+\frac{\lambda(\lambda-1) \mu}{z(z-1)(z-\lambda)}-\frac{t(t-1) H}{z(z-1)(z-t)}\right) y_{1}=0, \\
H= & \frac{1}{t(t-1)}\left[\lambda(\lambda-1)(\lambda-t) \mu^{2}-\left\{\theta_{0}(\lambda-1)(\lambda-t)+\theta_{1} \lambda(\lambda-t)\right.\right. \\
& \left.\left.+\left(\theta_{t}-1\right) \lambda(\lambda-1)\right\} \mu+\kappa_{1}\left(\kappa_{2}+1\right)(\lambda-t)\right],
\end{aligned}
$$


which we denote by $D_{y_{1}}\left(\theta_{0}, \theta_{1}, \theta_{t}, \theta_{\infty} ; \lambda, \mu\right)$. This equation has regular singularities at $z=$ $0,1, t, \lambda, \infty$. Exponents of the singularity $z=\lambda$ are 0,2 , and it is apparent (non-logarithmic) singularity. Note that the differential equations

$$
\frac{d \lambda}{d t}=\frac{\partial H}{\partial \mu}, \quad \frac{d \mu}{d t}=-\frac{\partial H}{\partial \lambda}
$$

describe the condition for monodromy preserving deformation of equation (2.3) with respect to the variable $t$. By eliminating the variable $\mu$ in equation (2.11), we have the sixth Painlevé equation on the variable $\lambda$ (see equation (1.2)). See [20] on equations (2.3), (2.10) and (2.11).

We consider realization of the Fuchsian system (equation (2.1)) for the case $\lambda=0,1, t, \infty$ in the appendix.

\section{The space of initial conditions for the sixth Painlevé equation and Heun's equation}

In this section, we introduce the space of initial conditions for the sixth Painlevé equation, restrict the variables of the space of initial conditions $E(t)$ to certain lines, and we obtain Heun's equation.

The space of initial conditions was introduced by Okamoto [18], which is a suitable defining variety for the set of solutions to the Painlevé system. In [22], Shioda and Takano studied the space of initial conditions further for the sixth Painlevé system (equation (2.11)) to study roles of holomorphy on the Hamiltonian. It was also constructed as a moduli space of parabolic connections by Inaba, Iwasaki and Saito $[8,9]$. Here we adopt the coordinate of initial coordinate by Shioda and Takano [22] (see also [33]). The space of initial condition $E(t)$ is defined by patching six copies

$$
\begin{array}{lll}
U_{0}=\left\{\left(q_{0}, p_{0}\right)\right\}, & U_{1}=\left\{\left(q_{1}, p_{1}\right)\right\}, & U_{2}=\left\{\left(q_{2}, p_{2}\right)\right\}, \\
U_{3}=\left\{\left(q_{3}, p_{3}\right)\right\}, & U_{4}=\left\{\left(q_{4}, p_{4}\right)\right\}, & U_{\infty}=\left\{\left(q_{\infty}, p_{\infty}\right)\right\},
\end{array}
$$

of $\mathbb{C}^{2}$ for fixed $\left(t ; \theta_{0}, \theta_{1}, \theta_{t}, \theta_{\infty}\right)$, and the rule of patching is defined by

$$
\begin{array}{lll}
q_{0} q_{\infty}=1, & q_{0} p_{0}+q_{\infty} p_{\infty}=-\kappa_{1}, & \left(U_{0} \cap U_{\infty}\right), \\
q_{0} p_{0}+q_{1} p_{1}=\theta_{0}, & p_{0} p_{1}=1, & \left(U_{0} \cap U_{1}\right), \\
\left(q_{0}-1\right) p_{0}+q_{2} p_{2}=\theta_{1}, & p_{0} p_{2}=1, & \left(U_{0} \cap U_{2}\right), \\
\left(q_{0}-t\right) p_{0}+q_{3} p_{3}=\theta_{t}, & p_{0} p_{3}=1, & \left(U_{0} \cap U_{3}\right), \\
q_{\infty} p_{\infty}+q_{4} p_{4}=1-\theta_{\infty}, & p_{\infty} p_{4}=1, & \left(U_{\infty} \cap U_{4}\right),
\end{array}
$$

The variables $(\lambda, \mu)$ of the sixth Painlevé system (see equation (2.11)) are realized as $q_{0}=\lambda$, $p_{0}=\mu$ in $U_{0}$.

We define complex lines in the space of initial conditions as follows:

$$
\begin{array}{ll}
L_{0}=\left\{\left(0, p_{0}\right)\right\} \subset U_{0}, & L_{1}=\left\{\left(1, p_{0}\right)\right\} \subset U_{0}, \\
L_{t}=\left\{\left(t, p_{0}\right)\right\} \subset U_{0}, & L_{\infty}=\left\{\left(0, p_{\infty}\right)\right\} \subset U_{\infty}, \\
L_{0}^{*}=\left\{\left(q_{1}, 0\right)\right\} \subset U_{1}, & L_{1}^{*}=\left\{\left(q_{2}, 0\right)\right\} \subset U_{2}, \\
L_{t}^{*}=\left\{\left(q_{3}, 0\right)\right\} \subset U_{3}, & L_{\infty}^{*}=\left\{\left(q_{4}, 0\right)\right\} \subset U_{4} .
\end{array}
$$

Set

$$
U_{0}^{q_{0} \neq 0,1, t}=U_{0} \backslash\left(L_{0} \cup L_{1} \cup L_{t}\right) .
$$


Then the space of initial conditions $E(t)$ is a direct sum of the sets $U_{0}^{q_{0} \neq 0,1, t}, L_{0}, L_{1}, L_{t}, L_{\infty}$, $L_{0}^{*}, L_{1}^{*}, L_{t}^{*}, L_{\infty}^{*}$. If $(\lambda, \mu) \in U_{0}^{q_{0} \neq 0,1, t}$, then $\lambda \neq 0,1, t, \infty$ and equation (2.10) has five regular singularities $\{0,1, t, \lambda, \infty\}$.

Although equation (2.6) was considered on the set $U_{0}^{q_{0} \neq 0,1, t}$, we may consider realization of a second-order differential equation as equation (2.10) on the space of initial conditions $E(t)$. On the lines $L_{0}, L_{1}, L_{t}$, equation (2.10) is realized by setting $\lambda=0,1, t$, and the equation is written in the form of Heun's equation

$$
\begin{aligned}
& \frac{d^{2} y_{1}}{d z^{2}}+\left(\frac{-\theta_{0}}{z}+\frac{1-\theta_{1}}{z-1}+\frac{1-\theta_{t}}{z-t}\right) \frac{d y_{1}}{d z}+\frac{\kappa_{1}\left(\kappa_{2}+1\right) z+t \theta_{0} \mu}{z(z-1)(z-t)} y_{1}=0, \\
& \frac{d^{2} y_{1}}{d z^{2}}+\left(\frac{1-\theta_{0}}{z}+\frac{-\theta_{1}}{z-1}+\frac{1-\theta_{t}}{z-t}\right) \frac{d y_{1}}{d z}+\frac{\kappa_{1}\left(\kappa_{2}+1\right)(z-1)+(1-t) \theta_{1} \mu}{z(z-1)(z-t)} y_{1}=0, \\
& \frac{d^{2} y_{1}}{d z^{2}}+\left(\frac{1-\theta_{0}}{z}+\frac{1-\theta_{1}}{z-1}+\frac{-\theta_{t}}{z-t}\right) \frac{d y_{1}}{d z}+\frac{\kappa_{1}\left(\kappa_{2}+1\right)(z-t)+t(t-1) \theta_{t} \mu}{z(z-1)(z-t)} y_{1}=0,
\end{aligned}
$$

respectively. Note that if $\theta_{0} \theta_{1} \theta_{t} \neq 0$ then we can realize all values of accessory parameter as varying $\mu$. For the case $\theta_{0} \theta_{1} \theta_{t}=0$, we should consider other realizations.

To realize equation (2.10) on the line $L_{0}^{*}$, we change the variables $(\lambda, \mu)$ into the ones $\left(q_{1}, p_{1}\right)$ on equation (2.10) by applying relations $\lambda \mu+q_{1} p_{1}=\theta_{0}, \mu p_{1}=1$. Then we have

$$
\begin{aligned}
\frac{d^{2} y_{1}}{d z^{2}} & +\left(\frac{1-\theta_{0}}{z}+\frac{1-\theta_{1}}{z-1}+\frac{1-\theta_{t}}{z-t}-\frac{1}{z+p_{1}\left(p_{1} q_{1}-\theta_{0}\right)}\right) \frac{d y_{1}}{d z} \\
& +\frac{\kappa_{1}\left(\kappa_{2}+1\right) z^{2}+\left(t q_{1}-\theta_{0}\left(\theta_{t}+t \theta_{1}+p_{1} \mathrm{pol}_{1}\right) z+\left(p_{1} q_{1}-\theta_{0}\right)\left(-t-p_{1} \mathrm{pol}_{2}\right)\right.}{z(z-1)(z-t)\left(z+p_{1}\left(p_{1} q_{1}-\theta_{0}\right)\right)} y_{1}=0,
\end{aligned}
$$

where $\mathrm{pol}_{1}$ and $\mathrm{pol}_{2}$ are polynomials in $p_{1}, q_{1}, t, \theta_{0}, \theta_{1}, \theta_{t}, \theta_{\infty}$. By setting $p_{1}=0$, we obtain

$$
\begin{aligned}
\frac{d^{2} y_{1}}{d z^{2}} & +\left(\frac{-\theta_{0}}{z}+\frac{1-\theta_{1}}{z-1}+\frac{1-\theta_{t}}{z-t}\right) \frac{d y_{1}}{d z} \\
& +\frac{\kappa_{1}\left(\kappa_{2}+1\right) z^{2}+\left(t q_{1}-\theta_{0}\left(\theta_{t}+t \theta_{1}\right)\right) z+t \theta_{0}}{z^{2}(z-1)(z-t)} y_{1}=0 .
\end{aligned}
$$

Since the exponents of equation (3.7) at $z=0$ are 1 and $\theta_{0}$, we consider gauge-transformation $v_{1}=z^{-1} y_{1}$ to obtain Heun's equation, and we have

$$
\begin{aligned}
& \frac{d^{2} v_{1}}{d z^{2}}+\left(\frac{2-\theta_{0}}{z}+\frac{1-\theta_{1}}{z-1}+\frac{1-\theta_{t}}{z-t}\right) \frac{d v_{1}}{d z}+\frac{\left(\kappa_{1}+1\right)\left(\kappa_{2}+2\right) z-q}{z(z-1)(z-t)} v_{1}=0 \\
& q=-t q_{1}+\left(\theta_{0}-1\right)\left\{t\left(\theta_{1}-1\right)+\theta_{t}-1\right\}
\end{aligned}
$$

To realize the second-order Fuchsian equation on the line $L_{1}^{*}$, we change the variables $(\lambda, \mu)$ into the ones $\left(q_{2}, p_{2}\right)$, substitute $p_{2}=0$ into equation (2.10) and set $v_{1}=(z-1)^{-1} y_{1}$. Then $v_{1}$ satisfies the following equation;

$$
\begin{aligned}
& \frac{d^{2} v_{1}}{d z^{2}}+\left(\frac{1-\theta_{0}}{z}+\frac{2-\theta_{1}}{z-1}+\frac{1-\theta_{t}}{z-t}\right) \frac{d v_{1}}{d z}+\frac{\left(\kappa_{1}+1\right)\left(\kappa_{2}+2\right)(z-1)-q}{z(z-1)(z-t)} v_{1}=0, \\
& q=(t-1) q_{2}-\left(\theta_{1}-1\right)\left\{(1-t)\left(\theta_{0}-1\right)+\theta_{t}-1\right\} .
\end{aligned}
$$

The second-order Fuchsian equation on the line $L_{t}^{*}$ is realized as

$$
\frac{d^{2} v_{1}}{d z^{2}}+\left(\frac{1-\theta_{0}}{z}+\frac{1-\theta_{1}}{z-1}+\frac{2-\theta_{t}}{z-t}\right) \frac{d v_{1}}{d z}+\frac{\left(\kappa_{1}+1\right)\left(\kappa_{2}+2\right)(z-t)-q}{z(z-1)(z-t)} v_{1}=0,
$$




$$
q=t(1-t) q_{3}-\left(\theta_{t}-1\right)\left((t-1)\left(\theta_{0}-1\right)+t\left(\theta_{1}-1\right)\right)
$$

by setting $p_{3}=0$ and $v_{1}=(z-t)^{-1} y_{1}$.

We investigate equation (2.10) on the line $L_{\infty}$. We change the variables $(\lambda, \mu)$ into the ones $\left(q_{\infty}, p_{\infty}\right)$ on equation (2.10) by applying relations $\lambda q_{\infty}=1, \lambda \mu+q_{\infty} p_{\infty}=-\kappa_{1}$, and substitute $q_{\infty}=0$. Then we have

$$
\begin{aligned}
& \frac{d^{2} y_{1}}{d z^{2}}+\left(\frac{1-\theta_{0}}{z}+\frac{1-\theta_{1}}{z-1}+\frac{1-\theta_{t}}{z-t}\right) \frac{d y_{1}}{d z}+\frac{\kappa_{1}\left(\kappa_{2}+2\right) z-q}{z(z-1)(z-t)} y_{1}=0 \\
& q=\left(\theta_{\infty}-1\right) p_{\infty}+\kappa_{1}\left(t\left(\kappa_{2}+\theta_{t}+1\right)+\kappa_{2}+\theta_{1}+1\right)
\end{aligned}
$$

Note that the exponents at $z=\infty$ are $\kappa_{1}$ and $\kappa_{2}+2$.

To realize equation (2.10) on the line $L_{\infty}^{*}$, we change the variables $(\lambda, \mu)$ into the ones $\left(q_{4}, p_{4}\right)$ on equation (2.10) by applying relations $\lambda q_{\infty}=1, \lambda \mu+q_{\infty} p_{\infty}=-\kappa_{1}, q_{\infty} p_{\infty}+q_{4} p_{4}=1-\theta_{\infty}$, $p_{\infty} p_{4}=1$, substitute $p_{4}=0$. We obtain

$$
\begin{aligned}
& \frac{d^{2} y_{1}}{d z^{2}}+\left(\frac{1-\theta_{0}}{z}+\frac{1-\theta_{1}}{z-1}+\frac{1-\theta_{t}}{z-t}\right) \frac{d y_{1}}{d z}+\frac{\left(\kappa_{1}+1\right)\left(\kappa_{2}+1\right) z-q}{z(z-1)(z-t)} y_{1}=0 \\
& q=-q_{4}+\left(\kappa_{2}+1\right)\left(t\left(\kappa_{1}+\theta_{t}\right)+\kappa_{1}+\theta_{1}\right) .
\end{aligned}
$$

The exponents at $z=\infty$ are $\kappa_{1}+1$ and $\kappa_{2}+1$, which are different from the case of the line $L_{\infty}$.

The Fuchsian system $D_{Y}\left(\theta_{0}, \theta_{1}, \theta_{t}, \theta_{\infty} ; \lambda, \mu ; k\right)$ is originally defined on the set $U_{0}^{q_{0} \neq 0,1, t}$. We try to consider realization of Fuchsian system (equation (2.1)) on the lines $L_{0}, L_{0}^{*}, L_{1}, L_{1}^{*}, L_{t}$, $L_{t}^{*}, L_{\infty}, L_{\infty}^{*}$ in the appendix.

\section{Middle convolution}

First, we review an algebraic analogue of Katz' middle convolution functor developed by Dettweiler and Reiter [2,3], which we restrict to the present setting. Let $A_{0}, A_{1}, A_{t}$ be matrices in $\mathbb{C}^{2 \times 2}$. For $\nu \in \mathbb{C}$, we define the convolution matrices $B_{0}, B_{1}, B_{t} \in \mathbb{C}^{6 \times 6}$ as follows:

$$
\begin{array}{rlrl}
B_{0} & =\left(\begin{array}{ccc}
A_{0}+\nu & A_{1} & A_{t} \\
0 & 0 & 0 \\
0 & 0 & 0
\end{array}\right), & B_{1}=\left(\begin{array}{ccc}
0 & 0 & 0 \\
A_{0} & A_{1}+\nu & A_{t} \\
0 & 0 & 0
\end{array}\right), \\
B_{t} & =\left(\begin{array}{ccc}
0 & 0 & 0 \\
0 & 0 & 0 \\
A_{0} & A_{1} & A_{t}+\nu
\end{array}\right) .
\end{array}
$$

Let $z \in \mathbb{C} \backslash\{0,1, t\}, \gamma_{p}(p \in \mathbb{C})$ be a cycle in $\mathbb{C} \backslash\{0,1, t, z\}$ turning the point $w=p$ anti-clockwise whose fixed base point is $o \in \mathbb{C} \backslash\{0,1, t, z\}$, and $\left[\gamma_{p}, \gamma_{p^{\prime}}\right]=\gamma_{p} \gamma_{p^{\prime}} \gamma_{p}^{-1} \gamma_{p^{\prime}}^{-1}$ be the Pochhammer contour.

Proposition 1 ([3]). Assume that $Y={ }^{t}\left(y_{1}(z), y_{2}(z)\right)$ is a solution to the system of differential equations

$$
\frac{d Y}{d z}=\left(\frac{A_{0}}{z}+\frac{A_{1}}{z-1}+\frac{A_{t}}{z-t}\right) Y
$$


For $p \in\{0,1, t, \infty\}$, the function

$$
U=\left(\begin{array}{l}
\int_{\left[\gamma_{z}, \gamma_{p}\right]} w^{-1} y_{1}(w)(z-w)^{\nu} d w \\
\int_{\left[\gamma_{z}, \gamma_{p}\right]} w^{-1} y_{2}(w)(z-w)^{\nu} d w \\
\int_{\left[\gamma_{z}, \gamma_{p}\right]}(w-1)^{-1} y_{1}(w)(z-w)^{\nu} d w \\
\int_{\left[\gamma_{z}, \gamma_{p}\right]}(w-1)^{-1} y_{2}(w)(z-w)^{\nu} d w \\
\int_{\left[\gamma_{z}, \gamma_{p}\right]}(w-t)^{-1} y_{1}(w)(z-w)^{\nu} d w \\
\int_{\left[\gamma_{z}, \gamma_{p}\right]}(w-t)^{-1} y_{2}(w)(z-w)^{\nu} d w
\end{array}\right),
$$

satisfies the system of differential equations

$$
\frac{d U}{d z}=\left(\frac{B_{0}}{z}+\frac{B_{1}}{z-1}+\frac{B_{t}}{z-t}\right) U
$$

We set

$$
\begin{aligned}
& \mathcal{L}_{0}=\left(\begin{array}{c}
\operatorname{Ker}\left(A_{0}\right) \\
0 \\
0
\end{array}\right), \quad \mathcal{L}_{1}=\left(\begin{array}{c}
0 \\
\operatorname{Ker}\left(A_{1}\right) \\
0
\end{array}\right), \quad \mathcal{L}_{t}=\left(\begin{array}{c}
0 \\
0 \\
\operatorname{Ker}\left(A_{t}\right)
\end{array}\right), \\
& \mathcal{L}=\mathcal{L}_{0} \oplus \mathcal{L}_{1} \oplus \mathcal{L}_{t}, \quad \mathcal{K}=\operatorname{Ker}\left(B_{0}\right) \cap \operatorname{Ker}\left(B_{1}\right) \cap \operatorname{Ker}\left(B_{t}\right),
\end{aligned}
$$

where $\mathcal{L}_{0}, \mathcal{L}_{1}, \mathcal{L}_{t}, \mathcal{K} \subset \mathbb{C}^{6}$ and 0 in equation (4.3) means the zero vector in $\mathbb{C}^{2}$. We fix an isomorphism between $\mathbb{C}^{6} /(\mathcal{K}+\mathcal{L})$ and $\mathbb{C}^{m}$ for some $m$. A tuple of matrices $m c_{\nu}(A)=$ $\left(\tilde{B}_{0}, \tilde{B}_{1}, \tilde{B}_{t}\right)$, where $\tilde{B}_{p}(p=0,1, t)$ is induced by the action of $B_{p}$ on $\mathbb{C}^{m} \simeq \mathbb{C}^{6} /(\mathcal{K}+\mathcal{L})$, is called an additive version of the middle convolution of $\left(A_{0}, A_{1}, A_{t}\right)$ with the parameter $\nu$. Let $A_{0}, A_{1}, A_{t}$ be the matrices defined by equation (2.5). Then it is shown that if $\nu=0, \kappa_{1}, \kappa_{2}$ $\left(\right.$ resp. $\left.\nu \neq 0, \kappa_{1}, \kappa_{2}\right)$ then $\operatorname{dim} \mathbb{C}^{6} /(\mathcal{K}+\mathcal{L})=2\left(\right.$ resp. $\left.\operatorname{dim} \mathbb{C}^{6} /(\mathcal{K}+\mathcal{L})=3\right)$. If $\nu=0$, then the middle convolution is identity (see [3]). Hence the middle convolutions for two cases $\nu=\kappa_{1}, \kappa_{2}$ may lead to non-trivial transformations on the $2 \times 2$ Fuchsian system with four singularities $\{0,1, t, \infty\}$. Filipuk [5] obtained that the middle convolution for the case $\nu=\kappa_{1}$ induce an Okamoto's transformation of the sixth Painlevé system.

We now calculate explicitly the Fuchsian system of differential equations determined by the middle convolution for the case $\nu=\kappa_{2}$. Note that the following calculation is analogous to the one in [30] for the case $\nu=\kappa_{1}$. If $\nu=\kappa_{2}$, then the spaces $\mathcal{L}_{0}, \mathcal{L}_{1}, \mathcal{L}_{t}, \mathcal{K}$ are written as

$$
\mathcal{L}_{0}=\mathbb{C}\left(\begin{array}{c}
w_{0} \\
u_{0}+\theta_{0} \\
0 \\
0 \\
0 \\
0
\end{array}\right), \quad \mathcal{L}_{1}=\mathbb{C}\left(\begin{array}{c}
0 \\
0 \\
w_{1} \\
u_{1}+\theta_{1} \\
0 \\
0
\end{array}\right), \quad \mathcal{L}_{t}=\mathbb{C}\left(\begin{array}{c}
0 \\
0 \\
0 \\
0 \\
w_{t} \\
u_{t}+\theta_{t}
\end{array}\right), \quad \mathcal{K}=\mathbb{C}\left(\begin{array}{l}
0 \\
1 \\
0 \\
1 \\
0 \\
1
\end{array}\right)
$$


Set

$$
\begin{aligned}
& S=\left(\begin{array}{cccccc}
0 & 0 & 0 & w_{0} & 0 & 0 \\
0 & 0 & 1 & u_{0}+\theta_{0} & 0 & 0 \\
0 & 0 & 0 & 0 & w_{1} & 0 \\
s_{41} & s_{42} & 1 & 0 & u_{1}+\theta_{1} & 0 \\
0 & 0 & 0 & 0 & 0 & w_{t} \\
s_{61} & s_{62} & 1 & 0 & 0 & u_{t}+\theta_{t}
\end{array}\right), \\
& s_{41}=\frac{\mu(\lambda-t)+\kappa_{1}}{k \kappa_{1}}, \quad s_{61}=\frac{t\left(\mu(\lambda-1)+\kappa_{1}\right)}{k \kappa_{1}}, \quad s_{42}=\frac{\tilde{\lambda}-\lambda}{\lambda(\lambda-1) \kappa_{2}}, \\
& s_{62}=\frac{t(\tilde{\lambda}-\lambda)}{\lambda(\lambda-t) \kappa_{2}}, \quad \tilde{\lambda}=\lambda-\frac{\kappa_{2}}{\mu-\frac{\theta_{0}}{\lambda}-\frac{\theta_{1}}{(\lambda-1)}-\frac{\theta_{t}}{(\lambda-t)}},
\end{aligned}
$$

and $\tilde{U}=S^{-1} U$, where $U$ is a solution to equation (4.2). Then $\operatorname{det} U=k^{2}(\tilde{\lambda}-\lambda) /\left(t(1-t) \kappa_{2}\right)$ and $\tilde{U}$ satisfies

$$
\frac{d \tilde{U}}{d z}=\left(\begin{array}{cccccc}
b_{11}(z) & b_{12}(z) & 0 & 0 & 0 & 0 \\
b_{21}(z) & b_{22}(z) & 0 & 0 & 0 & 0 \\
\frac{-\left(u_{0}+\theta_{0}\right) \theta_{\infty} t}{k \kappa_{1} \lambda z} & \frac{\tilde{\lambda}}{\lambda z} & 0 & 0 & 0 & 0 \\
\frac{t}{k \lambda z} & 0 & 0 & \frac{\kappa_{2}}{z} & 0 & 0 \\
\frac{1-t}{k(\lambda-1)(z-1)} & 0 & 0 & 0 & \frac{\kappa_{2}}{z-1} & 0 \\
\frac{t(1-t)}{k(\lambda-t)(z-t)} & 0 & 0 & 0 & 0 & \frac{\kappa_{2}}{z-t}
\end{array}\right) \tilde{U}
$$

where $b_{11}(z), \ldots, b_{22}(z)$ are calculated such that the system of differential equation

$$
\frac{d \tilde{Y}}{d z}=\left(\begin{array}{cc}
b_{11}(z) & b_{12}(z) \\
b_{21}(z) & b_{22}(z)
\end{array}\right) \tilde{Y}, \quad \tilde{Y}=\left(\begin{array}{c}
\tilde{u}_{1}(z) \\
\tilde{u}_{2}(z)
\end{array}\right)
$$

coincides with the Fuchsian system $D_{Y}\left(\tilde{\theta}_{0}, \tilde{\theta}_{1}, \tilde{\theta}_{t}, \tilde{\theta}_{\infty} ; \tilde{\lambda}, \tilde{\mu} ; \tilde{k}\right)$ (see equation $(2.9)$ ), where

$$
\begin{aligned}
& \tilde{\theta}_{0}=\frac{\theta_{0}-\theta_{1}-\theta_{t}-\theta_{\infty}}{2}, \quad \tilde{\theta}_{1}=\frac{-\theta_{0}+\theta_{1}-\theta_{t}-\theta_{\infty}}{2}, \quad \tilde{\theta}_{t}=\frac{-\theta_{0}-\theta_{1}+\theta_{t}-\theta_{\infty}}{2}, \\
& \tilde{\theta}_{\infty}=\frac{-\theta_{0}-\theta_{1}-\theta_{t}+\theta_{\infty}}{2}, \quad \tilde{\lambda}=\lambda-\frac{\kappa_{2}}{\mu-\frac{\theta_{0}}{\lambda}-\frac{\theta_{1}}{\lambda-1}-\frac{\theta_{t}}{\lambda-t}}, \\
& \tilde{\mu}=\frac{\kappa_{2}+\theta_{0}}{\tilde{\lambda}}+\frac{\kappa_{2}+\theta_{1}}{\tilde{\lambda}-1}+\frac{\kappa_{2}+\theta_{t}}{\tilde{\lambda}-t}+\frac{\kappa_{2}}{\lambda-\tilde{\lambda}}, \quad \tilde{k}=k .
\end{aligned}
$$

The functions $\tilde{u}_{1}(z)$ and $\tilde{u}_{2}(z)$ are expressed as

$$
\begin{aligned}
\tilde{u}_{1}(z)= & \left(u_{0}+\theta_{0}\right) u_{1}(z)-\frac{k \lambda}{t} u_{2}(z)+\left(u_{1}+\theta_{1}\right) u_{3}(z) \\
& +\frac{k(\lambda-1)}{t-1} u_{4}(z)+\left(u_{t}+\theta_{t}\right) u_{5}(z)+\frac{k(\lambda-t)}{t(1-t)} u_{6}(z), \\
\tilde{u}_{2}(z)= & \frac{\kappa_{2} \lambda(\lambda-1)(\lambda-t)}{\kappa_{1}(\lambda-\tilde{\lambda})}\left(\frac{\left(\lambda \mu+\kappa_{1}\right)\left(u_{0}+\theta_{0}\right)}{k \lambda} u_{1}(z)-\frac{\lambda \mu+\kappa_{1}}{t} u_{2}(z)\right. \\
& +\frac{\left((\lambda-1) \mu+\kappa_{1}\right)\left(u_{1}+\theta_{1}\right)}{k(\lambda-1)} u_{3}(z)+\frac{(\lambda-1) \mu+\kappa_{1}}{t-1} u_{4}(z) \\
& \left.+\frac{\left((\lambda-t) \mu+\kappa_{1}\right)\left(u_{t}+\theta_{t}\right)}{k(\lambda-t)} u_{5}(z)+\frac{(\lambda-t) \mu+\kappa_{1}}{t(1-t)} u_{6}(z)\right) .
\end{aligned}
$$

Combining Proposition 1 with equation (4.6) and setting $\tilde{y}_{1}(z)=\tilde{u}_{1}(z), \tilde{y}_{2}(z)=\tilde{u}_{2}(z)$, we have the following theorem by means of a straightforward calculation: 
Theorem 1. Set $\kappa_{1}=\left(\theta_{\infty}-\theta_{0}-\theta_{1}-\theta_{t}\right) / 2$ and $\kappa_{2}=-\left(\theta_{\infty}+\theta_{0}+\theta_{1}+\theta_{t}\right) / 2$. If $y_{1}(z)$ is a solution to the Fuchsian equation $D_{y_{1}}\left(\theta_{0}, \theta_{1}, \theta_{t}, \theta_{\infty} ; \lambda, \mu\right)$, then the function $\tilde{Y}={ }^{t}\left(\tilde{y}_{1}(z), \tilde{y}_{2}(z)\right)$ defined by

$$
\begin{aligned}
& \tilde{y}_{1}(z)=\int_{\left[\gamma_{z}, \gamma_{p}\right]} \frac{d y_{1}(w)}{d w}(z-w)^{\kappa_{2}} d w \\
& \tilde{y}_{2}(z)=\frac{\kappa_{2} \lambda(\lambda-1)(\lambda-t)}{k(\lambda-\tilde{\lambda})} \int_{\left[\gamma_{z}, \gamma_{p}\right]}\left\{\left(\frac{d y_{1}(w)}{d w}-\mu y_{1}(w)\right) \frac{1}{\lambda-w}+\frac{\mu}{\kappa_{1}} \frac{d y_{1}(w)}{d w}\right\}(z-w)^{\kappa_{2}} d w,
\end{aligned}
$$

satisfies the Fuchsian system $D_{Y}\left(\kappa_{2}+\theta_{0}, \kappa_{2}+\theta_{1}, \kappa_{2}+\theta_{t}, \kappa_{2}+\theta_{\infty} ; \tilde{\lambda}, \tilde{\mu} ; k\right)$ for $p \in\{0,1, t, \infty\}$, where

$$
\tilde{\lambda}=\lambda-\frac{\kappa_{2}}{\mu-\frac{\theta_{0}}{\lambda}-\frac{\theta_{1}}{\lambda-1}-\frac{\theta_{t}}{\lambda-t}}, \quad \tilde{\mu}=\frac{\kappa_{2}+\theta_{0}}{\tilde{\lambda}}+\frac{\kappa_{2}+\theta_{1}}{\tilde{\lambda}-1}+\frac{\kappa_{2}+\theta_{t}}{\tilde{\lambda}-t}+\frac{\kappa_{2}}{\lambda-\tilde{\lambda}} .
$$

Since

$$
\begin{aligned}
0 & =\int_{\left[\gamma_{z}, \gamma_{p}\right]} \frac{d}{d w}\left(y_{1}(w)(z-w)^{\kappa_{2}}\right) d w \\
& =\int_{\left[\gamma_{z}, \gamma_{p}\right]} \frac{d y_{1}(w)}{d w}(z-w)^{\kappa_{2}} d w+\kappa_{2} \int_{\left[\gamma_{z}, \gamma_{p}\right]} y_{1}(w)(z-w)^{\kappa_{2}-1} d w
\end{aligned}
$$

we have

Proposition 2 ([17]). If $y_{1}(z)$ is a solution to $D_{y_{1}}\left(\theta_{0}, \theta_{1}, \theta_{t}, \theta_{\infty} ; \lambda, \mu\right)$, then the function

$$
\tilde{y}(z)=\int_{\left[\gamma_{z}, \gamma_{p}\right]} y_{1}(w)(z-w)^{\kappa_{2}-1} d w
$$

satisfies $D_{y_{1}}\left(\kappa_{2}+\theta_{0}, \kappa_{2}+\theta_{1}, \kappa_{2}+\theta_{t}, \kappa_{2}+\theta_{\infty} ; \tilde{\lambda}, \tilde{\mu}\right)$ for $p \in\{0,1, t, \infty\}$, where $\tilde{\lambda}$ and $\tilde{\mu}$ are defined in equation (4.8).

Note that this proposition was obtained by Novikov [17] by another method. Kazakov and Slavyanov [14] essentially obtained this proposition by investigating Euler transformation of $2 \times 2$ Fuchsian systems with singularities $\{0,1, t, \infty\}$ which are realized differently from $D_{Y}\left(\theta_{0}, \theta_{1}, \theta_{t}, \theta_{\infty} ; \lambda, \mu, k\right)$.

Let us recall the symmetry of the sixth Painlevé equation. It was essentially established by Okamoto [19] that the sixth Painlevé equation has symmetry of the affine Weyl group $W\left(D_{4}^{(1)}\right)$. More precisely, the sixth Painlevé system is invariant under the following transformations, which are involutive and satisfy Coxeter relations attached to the Dynkin diagram of type $D_{4}^{(1)}$, i.e. $\left(s_{i}\right)^{2}=1(i=0,1,2,3,4), s_{j} s_{k}=s_{k} s_{j}(j, k \in\{0,1,3,4\}), s_{j} s_{2} s_{j}=s_{2} s_{j} s_{2}(j=0,1,3,4)$ :

\begin{tabular}{|c||cccc|cc|c|}
\hline & $\theta_{t}$ & $\theta_{\infty}$ & $\theta_{1}$ & $\theta_{0}$ & $\lambda$ & $\mu$ & $t$ \\
\hline$s_{0}$ & $-\theta_{t}$ & $\theta_{\infty}$ & $\theta_{1}$ & $\theta_{0}$ & $\lambda$ & $\mu-\frac{\theta_{t}}{\lambda-t}$ & $t$ \\
$s_{1}$ & $\theta_{t}$ & $2-\theta_{\infty}$ & $\theta_{1}$ & $\theta_{0}$ & $\lambda$ & $\mu$ & $t$ \\
$s_{2}$ & $\kappa_{1}+\theta_{t}$ & $-\kappa_{2}$ & $\kappa_{1}+\theta_{1}$ & $\kappa_{1}+\theta_{0}$ & $\lambda+\frac{\kappa_{1}}{\mu}$ & $\mu$ & $t$ \\
$s_{3}$ & $\theta_{t}$ & $\theta_{\infty}$ & $-\theta_{1}$ & $\theta_{0}$ & $\lambda$ & $\mu-\frac{\theta_{1}}{\lambda-1}$ & $t$ \\
$s_{4}$ & $\theta_{t}$ & $\theta_{\infty}$ & $\theta_{1}$ & $-\theta_{0}$ & $\lambda$ & $\mu-\frac{\theta_{0}}{\lambda}$ & $t$ \\
\hline
\end{tabular}

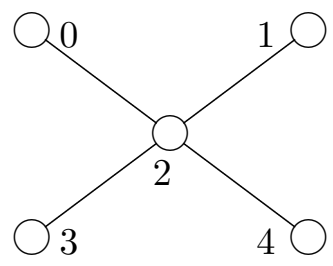

The map $\left(\theta_{0}, \theta_{1}, \theta_{t}, \theta_{\infty} ; \lambda, \mu\right) \mapsto\left(\tilde{\theta}_{0}, \tilde{\theta}_{1}, \tilde{\theta}_{t}, \tilde{\theta}_{\infty} ; \tilde{\lambda}, \tilde{\mu}\right)$ determined by equation (4.5) coincides with the composition map $s_{0} s_{3} s_{4} s_{2} s_{0} s_{3} s_{4}$, because

$$
\left(\theta_{0}, \theta_{1}, \theta_{t}, \theta_{\infty} ; \lambda, \mu\right) \stackrel{s_{0} s_{3} s_{4}}{\longmapsto}\left(-\theta_{0},-\theta_{1},-\theta_{t}, \theta_{\infty} ; \lambda, \mu-\frac{\theta_{0}}{\lambda}-\frac{\theta_{1}}{\lambda-1}-\frac{\theta_{t}}{\lambda-t}\right)
$$




$$
\begin{aligned}
& \stackrel{s_{2}}{\mapsto}\left(-\kappa_{2}-\theta_{0},-\kappa_{2}-\theta_{1},-\kappa_{2}-\theta_{t}, \kappa_{1} ; \tilde{\lambda}, \mu-\frac{\theta_{0}}{\lambda}-\frac{\theta_{1}}{\lambda-1}-\frac{\theta_{t}}{\lambda-t}\right) \\
& \stackrel{s_{0} s_{3} s_{4}}{\longmapsto}\left(\kappa_{2}+\theta_{0}, \kappa_{2}+\theta_{1}, \kappa_{2}+\theta_{t}, \kappa_{2}+\theta_{\infty} ; \tilde{\lambda}, \tilde{\mu}\right) .
\end{aligned}
$$

Therefore, if we know a solution to the Fuchsian system $D_{Y}\left(\theta_{0}, \theta_{1}, \theta_{t}, \theta_{\infty} ; \lambda, \mu ; k\right)$, then we have integral representations of solutions to the Fuchsian system $D_{Y}\left(\tilde{\theta}_{0}, \tilde{\theta}_{1}, \tilde{\theta}_{t}, \tilde{\theta}_{\infty} ; \tilde{\lambda}, \tilde{\mu} ; k\right)$ obtained by the transformation $s_{0} s_{3} s_{4} s_{2} s_{4} s_{3} s_{0}$. Note that the transformations $s_{i}(i=0,1,2,3,4)$ are extended to isomorphisms of the space of initial conditions $E(t)$.

We recall the middle convolution for the case $\nu=\kappa_{1}$.

Proposition 3 ([30, Proposition 3.2]). If $Y={ }^{t}\left(y_{1}(z), y_{2}(z)\right)$ is a solution to the Fuchsian system $D_{Y}\left(\theta_{0}, \theta_{1}, \theta_{t}, \theta_{\infty} ; \lambda, \mu ; k\right)$ (see equation $\left.(2.9)\right)$, then the function $\tilde{Y}={ }^{t}\left(\tilde{y}_{1}(z), \tilde{y}_{2}(z)\right)$ defined by

$$
\begin{aligned}
& \tilde{y}_{1}(z)=\int_{\left[\gamma_{z}, \gamma_{p}\right]}\left\{\kappa_{1} y_{1}(w)+(w-\tilde{\lambda}) \frac{d y_{1}(w)}{d w}\right\} \frac{(z-w)^{\kappa_{1}}}{w-\lambda} d w, \\
& \tilde{y}_{2}(z)=\frac{-\theta_{\infty}}{\kappa_{2}} \int_{\left[\gamma_{z}, \gamma_{p}\right]} \frac{d y_{2}(w)}{d w}(z-w)^{\kappa_{1}} d w,
\end{aligned}
$$

satisfies the Fuchsian system $D_{Y}\left(\kappa_{1}+\theta_{0}, \kappa_{1}+\theta_{1}, \kappa_{1}+\theta_{t},-\kappa_{2} ; \lambda+\kappa_{1} / \mu, \mu ; k\right)$ for $p \in\{0,1, t, \infty\}$.

The parameters $\left(\kappa_{1}+\theta_{0}, \kappa_{1}+\theta_{1}, \kappa_{1}+\theta_{t},-\kappa_{2} ; \lambda+\kappa_{1} / \mu, \mu\right)$ are obtained from the parameters $\left(\theta_{0}, \theta_{1}, \theta_{t}, \theta_{\infty} ; \lambda, \mu\right)$ by applying the transformation $s_{2}$. Note that the relationship the transformation $s_{2}$ was obtained by Filipuk [5] explicitly (see also [7]), and Boalch [1] and Dettweiler and Reiter [4] also obtained results on the symmetry of the sixth Painlevé equation and the middle convolution.

\section{Middle convolution, integral transformations of Heun's equation and the space of initial conditions}

In this section, we investigating relationship among the middle convolution, integral transformations of Heun's equation and the space of initial conditions.

Kazakov and Slavyanov established an integral transformation on solutions to Heun's equation in [13], which we express in a slightly different form.

Theorem 2 ([13]). Set

$$
\begin{aligned}
& (\eta-\alpha)(\eta-\beta)=0, \quad \gamma^{\prime}=\gamma+1-\eta, \quad \delta^{\prime}=\delta+1-\eta, \quad \epsilon^{\prime}=\epsilon+1-\eta, \\
& \left\{\alpha^{\prime}, \beta^{\prime}\right\}=\{2-\eta,-2 \eta+\alpha+\beta+1\}, \\
& q^{\prime}=q+(1-\eta)(\epsilon+\delta t+(\gamma-\eta)(t+1)) .
\end{aligned}
$$

Let $v(w)$ be a solution to

$$
\frac{d^{2} v}{d w^{2}}+\left(\frac{\gamma^{\prime}}{w}+\frac{\delta^{\prime}}{w-1}+\frac{\epsilon^{\prime}}{w-t}\right) \frac{d v}{d w}+\frac{\alpha^{\prime} \beta^{\prime} w-q^{\prime}}{w(w-1)(w-t)} v=0 .
$$

Then the function

$$
y(z)=\int_{\left[\gamma_{z}, \gamma_{p}\right]} v(w)(z-w)^{-\eta} d w
$$

is a solution to

$$
\frac{d^{2} y}{d z^{2}}+\left(\frac{\gamma}{z}+\frac{\delta}{z-1}+\frac{\epsilon}{z-t}\right) \frac{d y}{d z}+\frac{\alpha \beta z-q}{z(z-1)(z-t)} y=0
$$

for $p \in\{0,1, t, \infty\}$. 
Here we derive Theorem 2 by considering the limit $\lambda \rightarrow 0$ in Proposition 2. Let us recall notations in Proposition 2. We consider the limit $\lambda \rightarrow 0$ while fixing $\mu$ for the case $\theta_{0} \neq 0$ and $\theta_{0}+\kappa_{2} \neq 0$. Then we have $\tilde{\lambda} \rightarrow 0$ and $\tilde{\mu} \rightarrow\left(t \theta_{0} \mu+\kappa_{2}\left(t\left(\kappa_{1}+\theta_{t}\right)+\kappa_{1}+\theta_{1}\right)\right) /\left(t\left(\kappa_{2}+\theta_{0}\right)\right)$. Hence it follows from Proposition 2 and equation (3.4) that, if $y(z)$ satisfies

$$
\frac{d^{2} y(z)}{d z^{2}}+\left(\frac{-\theta_{0}}{z}+\frac{1-\theta_{1}}{z-1}+\frac{1-\theta_{t}}{z-t}\right) \frac{d y(z)}{d z}+\frac{\kappa_{1}\left(\kappa_{2}+1\right) z+t \theta_{0} \mu}{z(z-1)(z-t)} y(z)=0,
$$

then the function

$$
\tilde{y}(z)=\int_{\left[\gamma_{z}, \gamma_{p}\right]} y(w)(z-w)^{\kappa_{2}-1} d w,
$$

satisfies

$$
\begin{aligned}
\frac{d^{2} \tilde{y}(z)}{d z^{2}} & +\left(\frac{-\kappa_{2}-\theta_{0}}{z}+\frac{1-\kappa_{2}-\theta_{1}}{z-1}+\frac{1-\kappa_{2}-\theta_{t}}{z-t}\right) \frac{d \tilde{y}(z)}{d z} \\
& +\left(\frac{\theta_{\infty}\left(1-\kappa_{2}\right) z+t\left(\kappa_{2}+\theta_{0}\right) \frac{\left.t \theta_{0} \mu+\kappa_{2}\left(t\left(\kappa_{1}+\theta_{t}\right)+\kappa_{1}+\theta_{1}\right)\right)}{t\left(\kappa_{2}+\theta_{0}\right)}}{z(z-1)(z-t)}\right) \tilde{y}(z)=0 .
\end{aligned}
$$

By setting $\gamma=-\kappa_{2}-\theta_{0}, \delta=1-\kappa_{2}-\theta_{1}, \epsilon=1-\kappa_{2}-\theta_{t}, \alpha=\eta=1-\kappa_{2}, \beta=\theta_{\infty}$, $\left.q=-\left\{t \theta_{0} \mu+\kappa_{2}\left(t\left(\kappa_{1}+\theta_{t}\right)+\kappa_{1}+\theta_{1}\right)\right)\right\}$ and comparing with the standard form of Heun's equation (equation (1.1)), we recover Theorem 2. Note that we can obtain the formula corresponding to the case $\theta_{0}=0$ (resp. $\left.\theta_{0}+\kappa_{2}=0\right)$ by considering the limit $\theta_{0} \rightarrow 0$ (resp. $\theta_{0}+\kappa_{2} \rightarrow 0$ ).

The limit $\lambda \rightarrow 0$ while fixing $\mu$ implies the restriction of the coordinate $(\lambda, \mu)$ to the line $L_{0}$ in the space of initial conditions $E(t)$, and the line $L_{0}$ with the parameter $\left(\theta_{0}, \theta_{1}, \theta_{t}, \theta_{\infty} ; \lambda, \mu\right)$ is mapped to the line $L_{0}$ in the space of initial conditions with the parameter $\left(\kappa_{2}+\theta_{0}, \kappa_{2}+\theta_{1}, \kappa_{2}+\theta_{t}\right.$, $\left.\kappa_{2}+\theta_{\infty} ; \tilde{\lambda}, \tilde{\mu}\right)$ where $\tilde{\lambda}$ and $\tilde{\mu}$ are defined in equation (4.8), because $\tilde{\lambda} \rightarrow 0$ and $\tilde{\mu}$ converges by the limit. It follows from equations (5.4), (5.5), (5.6) that the integral transformation in Proposition 2 reproduces the integral transformation on Heun's equations in Theorem 2 by restricting to the line $L_{0}$. We can also establish that the line $L_{1}$ (resp. $L_{t}$ ) in the space of initial conditions with the parameter $\left(\theta_{0}, \theta_{1}, \theta_{t}, \theta_{\infty} ; \lambda, \mu\right)$ is mapped to the line $L_{1}$ (resp. $L_{t}$ ) with the parameter $\left(\kappa_{2}+\theta_{0}, \kappa_{2}+\theta_{1}, \kappa_{2}+\theta_{t}, \kappa_{2}+\theta_{\infty} ; \tilde{\lambda}, \tilde{\mu}\right)$ by taking the limit $\lambda \rightarrow 1$ (resp. $\lambda \rightarrow t$ ), and the integral transformation in Proposition 2 reproduces the integral transformation on Heun's equations in Theorem 2. We discuss the restriction of the map $\left(\theta_{0}, \theta_{1}, \theta_{t}, \theta_{\infty} ; \lambda, \mu\right) \mapsto$ $\left(\kappa_{2}+\theta_{0}, \kappa_{2}+\theta_{1}, \kappa_{2}+\theta_{t}, \kappa_{2}+\theta_{\infty} ; \tilde{\lambda}, \tilde{\mu}\right)$ to the line $L_{\infty}^{*}$. Let $\left(q_{4}, p_{4}\right)\left(\operatorname{resp} .\left(\tilde{q}_{4}, \tilde{p}_{4}\right)\right)$ be the coordinate of $U_{4}$ for the parameters $\left(\theta_{0}, \theta_{1}, \theta_{t}, \theta_{\infty} ; \lambda, \mu\right)$ (resp. $\left(\kappa_{2}+\theta_{0}, \kappa_{2}+\theta_{1}, \kappa_{2}+\theta_{t}, \kappa_{2}+\theta_{\infty} ; \tilde{\lambda}, \tilde{\mu}\right)$ (see equations (3.1), (3.2)). Then we can express $\tilde{q}_{4}$ and $\tilde{p}_{4}$ by the variables $q_{4}$ and $p_{4}$. By setting $p_{4}=0$, we have $\tilde{p}_{4}=0$ and $\tilde{q}_{4}=q_{4}-\kappa_{2}\left(t\left(\kappa_{1}+\theta_{t}-1\right)+\kappa_{1}+\theta_{1}-1\right)$. Hence the line $L_{\infty}^{*}$ with the parameter $\left(\theta_{0}, \theta_{1}, \theta_{t}, \theta_{\infty} ; \lambda, \mu\right)$ is mapped to the line $L_{\infty}^{*}$ with the parameter $\left(\kappa_{2}+\theta_{0}, \kappa_{2}+\theta_{1}, \kappa_{2}+\theta_{t}, \kappa_{2}+\theta_{\infty} ; \tilde{\lambda}, \tilde{\mu}\right)$. It follows from Proposition 2 that if $y_{1}(z)$ satisfies equation (3.11) then the function $\tilde{y}(z)$ defined by Proposition 2 satisfies Heun's equation with the parameters $\gamma=1-\theta_{0}-\kappa_{2}, \delta=1-\theta_{1}-\kappa_{2}, \epsilon=1-\theta_{t}-\kappa_{2}, \alpha=1-\kappa_{2}, \beta=1+\theta_{\infty}$, $\left.q=-q_{4}-(1+t) \kappa_{2}+\left(t\left(\kappa_{1}+\theta_{t}\right)+\kappa_{1}+\theta_{1}\right)\right)$, and the integral representation reproduces Theorem 2 by setting $\eta=\alpha=1-\kappa_{2}$. Therefore we have the following theorem:

Theorem 3. Let $X=L_{0}, L_{1}, L_{t}$ or $L_{\infty}^{*}$. By the map $s_{0} s_{3} s_{4} s_{2} s_{4} s_{3} s_{0}:\left(\theta_{0}, \theta_{1}, \theta_{t}, \theta_{\infty} ; \lambda, \mu\right) \mapsto$ $\left(\kappa_{2}+\theta_{0}, \kappa_{2}+\theta_{1}, \kappa_{2}+\theta_{t}, \kappa_{2}+\theta_{\infty} ; \tilde{\lambda}, \tilde{\mu}\right)$ where $\tilde{\lambda}$ and $\tilde{\mu}$ are defined in equation (4.8), the line $X$ in the space of initial conditions with the parameter $\left(\theta_{0}, \theta_{1}, \theta_{t}, \theta_{\infty} ; \lambda, \mu\right)$ is mapped to the line $X$ in the space of initial conditions with the parameter $\left(\kappa_{2}+\theta_{0}, \kappa_{2}+\theta_{1}, \kappa_{2}+\theta_{t}, \kappa_{2}+\theta_{\infty} ; \tilde{\lambda}, \tilde{\mu}\right)$ where $\tilde{\lambda}$ and $\tilde{\mu}$ are defined in equation (4.8), and the integral transformation in Proposition 2 determined by the middle convolution reproduces the integral transformation on Heun's equations in Theorem 2 by the restriction to the line $X$. 
Note that if $X=L_{0}^{*}, L_{1}^{*}, L_{t}^{*}$ or $L_{\infty}$ then the image of the line $X$ by the map $s_{0} s_{3} s_{4} s_{2} s_{4} s_{3} s_{0}$ may not included in $X$ with the parameter $\left(\kappa_{2}+\theta_{0}, \kappa_{2}+\theta_{1}, \kappa_{2}+\theta_{t}, \kappa_{2}+\theta_{\infty} ; \tilde{\lambda}, \tilde{\mu}\right)$.

We consider restrictions of the middle convolution for the case $\nu=\kappa_{1}$ (see Proposition 3) to lines in the space of initial conditions. We discuss the restriction of the map $\left(\theta_{0}, \theta_{1}, \theta_{t}, \theta_{\infty} ; \lambda, \mu\right)$ $\mapsto\left(\kappa_{1}+\theta_{0}, \kappa_{1}+\theta_{1}, \kappa_{1}+\theta_{t},-\kappa_{2} ; \lambda+\kappa_{1} / \mu, \mu\right)$ to the line $L_{0}^{*}$. Let $\left(q_{1}, p_{1}\right)$ (resp. $\left.\left(\tilde{q}_{1}, \tilde{p}_{1}\right)\right)$ be the coordinate of $U_{1}$ for the parameters $\left(\theta_{0}, \theta_{1}, \theta_{t}, \theta_{\infty} ; \lambda, \mu\right)\left(\operatorname{resp} .\left(\kappa_{1}+\theta_{0}, \kappa_{1}+\theta_{1}, \kappa_{1}+\theta_{t},-\kappa_{2} ; \lambda+\right.\right.$ $\left.\left.\kappa_{1} / \mu, \mu\right)\right)$. Then we can express $\tilde{q}_{1}$ and $\tilde{p}_{1}$ by the variables $q_{1}$ and $p_{1}$, and by setting $p_{1}=0$ we have $\tilde{p}_{1}=0$ and $\tilde{q}_{1}=q_{1}$. Let $y_{1}(z)$ be a solution to the Fuchsian equation $D_{y_{1}}\left(\theta_{0}, \theta_{1}, \theta_{t}, \theta_{\infty} ; \lambda, \mu\right)$ for the case $p_{1}=0$ and set $v_{1}(z)=z^{-1} y_{1}(z)$. Then $v_{1}(z)$ satisfies Heun's equation written as equation (3.8). On the case $p_{1}=0$, the integral representation (equation (4.11)) is written as

$$
\tilde{y}_{1}(z)=\int_{\left[\gamma_{z}, \gamma_{p}\right]}\left\{\kappa_{1} y_{1}(w)+w \frac{d y_{1}(w)}{d w}\right\} \frac{(z-w)^{\kappa_{1}}}{w} d w, \quad(p \in\{0,1, t, \infty\}) .
$$

We set $\tilde{v}_{1}(z)=z^{-1} \tilde{y}_{1}(z)$. By integration by parts we have

$$
\begin{aligned}
\tilde{v}_{1}(z) & =\frac{1}{z} \int_{\left[\gamma_{z}, \gamma_{p}\right]}\left\{\kappa_{1} v_{1}(w)(z-w)^{\kappa_{1}}+\frac{d\left(w v_{1}(w)\right)}{d w}(z-w)^{\kappa_{1}}\right\} d w \\
& =\frac{1}{z} \int_{\left[\gamma_{z}, \gamma_{p}\right]}\left\{\kappa_{1}(z-w) v_{1}(w)(z-w)^{\kappa_{1}-1}+\kappa_{1} w v_{1}(w)(z-w)^{\kappa_{1}-1}\right\} d w \\
& =\kappa_{1} \int_{\left[\gamma_{z}, \gamma_{p}\right]} v_{1}(w)(z-w)^{\kappa_{1}-1} d w .
\end{aligned}
$$

On the other hand, it follows from Proposition 3 and equation (3.8) that $\tilde{v}_{1}(z)$ satisfies Heun's equation with the parameters $\gamma=2-\theta_{0}-\kappa_{1}, \delta=1-\theta_{1}-\kappa_{1}, \epsilon=1-\theta_{t}-\kappa_{1}, \alpha=1-\kappa_{1}$, $\beta=2-\theta_{\infty}, q=-t q_{1}+\left(\kappa_{1}+\theta_{0}-1\right)\left\{t\left(\kappa_{1}+\theta_{1}-1\right)+\kappa_{1}+\theta_{t}-1\right\}$. Hence equation (5.7) reproduces Theorem 2 by setting $\eta=\alpha=1-\kappa_{1}$, We can also obtain similar results for $L_{1}^{*}, L_{t}^{*}$ and $L_{\infty}^{*}$. Therefore we have the following theorem:

Theorem 4. Let $X=L_{0}^{*}, L_{1}^{*}$, $L_{t}^{*}$ or $L_{\infty}^{*}$. By the map $s_{2}:\left(\theta_{0}, \theta_{1}, \theta_{t}, \theta_{\infty} ; \lambda, \mu\right) \mapsto\left(\kappa_{1}+\theta_{0}\right.$, $\left.\kappa_{1}+\theta_{1}, \kappa_{1}+\theta_{t},-\kappa_{2} ; \lambda+\kappa_{1} / \mu, \mu\right)$, the line $X$ in the space of initial conditions with the parameter $\left(\theta_{0}, \theta_{1}, \theta_{t}, \theta_{\infty} ; \lambda, \mu\right)$ is mapped to the line $X$ in the space of initial conditions with the parameter $\left(\kappa_{1}+\theta_{0}, \kappa_{1}+\theta_{1}, \kappa_{1}+\theta_{t},-\kappa_{2} ; \lambda+\kappa_{1} / \mu, \mu\right)$, and the integral transformation in Proposition 3 determined by the middle convolution reproduces the integral transformation on Heun's equations in Theorem 2 by the restriction to the line $X$.

Note that if $X=L_{0}, L_{1}, L_{t}$ or $L_{\infty}$ then the image of the line $X$ by the map $s_{2}$ may not included in $X$ with the parameter $\left(\kappa_{1}+\theta_{0}, \kappa_{1}+\theta_{1}, \kappa_{1}+\theta_{t},-\kappa_{2} ; \lambda+\kappa_{1} / \mu, \mu\right)$.

\section{Middle convolution for the case that the parameter is integer}

On the case $\kappa_{2} \in \mathbb{Z}$, the function in equation (4.10) containing the Pochhammer contour may be vanished, and we propose other expressions of solutions to Fuchsian equation $D_{y_{1}}\left(\kappa_{2}+\theta_{0}, \kappa_{2}+\theta_{1}\right.$, $\left.\kappa_{2}+\theta_{t}, \kappa_{2}+\theta_{\infty} ; \tilde{\lambda}, \tilde{\mu}\right)$ in use of solutions to $D_{y_{1}}\left(\theta_{0}, \theta_{1}, \theta_{t}, \theta_{\infty} ; \lambda, \mu\right)$.

We have the following proposition for the case $\kappa_{2} \in \mathbb{Z}_{<0}$,

Proposition 4. (i) Let $A_{0}, A_{1}, A_{t}$ be matrices in $\mathbb{C}^{2 \times 2}$, and let $B_{0}^{(\nu)}, B_{1}^{(\nu)}, B_{t}^{(\nu)} \in \mathbb{C}^{6 \times 6}$ be the matrices defined in equation (4.1) for $\nu \in \mathbb{C}$. Assume that $\nu \in \mathbb{Z}_{<0}$ and $Y={ }^{t}\left(y_{1}(z), y_{2}(z)\right)$ is a solution to the system of differential equations

$$
\frac{d Y}{d z}=\left(\frac{A_{0}}{z}+\frac{A_{1}}{z-1}+\frac{A_{t}}{z-t}\right) Y .
$$


Write $\nu=-1-n\left(n \in \mathbb{Z}_{\geq 0}\right)$. Then the function

$$
U=\left(\begin{array}{l}
(d / d z)^{n}\left(z^{-1} y_{1}(z)\right) \\
(d / d z)^{n}\left(z^{-1} y_{2}(z)\right) \\
(d / d z)^{n}\left((z-1)^{-1} y_{1}(z)\right) \\
(d / d z)^{n}\left((z-1)^{-1} y_{2}(z)\right) \\
(d / d z)^{n}\left((z-t)^{-1} y_{1}(z)\right) \\
(d / d z)^{n}\left((z-t)^{-1} y_{2}(z)\right)
\end{array}\right)
$$

satisfies the system of differential equations

$$
\frac{d U}{d z}=\left(\frac{B_{0}^{(-1-n)}}{z}+\frac{B_{1}^{(-1-n)}}{z-1}+\frac{B_{t}^{(-1-n)}}{z-t}\right) U .
$$

(ii) If $\kappa_{2} \in \mathbb{Z}_{<0}$ and $Y={ }^{t}\left(y_{1}(z), y_{2}(z)\right)$ is a solution to the Fuchsian system $D_{Y}\left(\theta_{0}, \theta_{1}, \theta_{t}, \theta_{\infty}\right.$; $\lambda, \mu ; k)$, then the function $\tilde{Y}={ }^{t}\left(\tilde{y}_{1}(z), \tilde{y}_{2}(z)\right)$ defined by

$$
\begin{aligned}
\tilde{y}_{1}(z)= & \left(\frac{d}{d z}\right)^{-\kappa_{2}} y_{1}(z), \\
\tilde{y}_{2}(z)= & \frac{\kappa_{2} \lambda(\lambda-1)(\lambda-t)}{k(\lambda-\tilde{\lambda})}\left[\left\{\left(\frac{d}{d z}\right)^{-\kappa_{2}} y_{1}(z)-\mu\left(\frac{d}{d z}\right)^{-\kappa_{2}-1} y_{1}(z)\right\} \frac{1}{\lambda-z}\right. \\
& \left.+\frac{\mu}{\kappa_{1}}\left(\frac{d}{d z}\right)^{-\kappa_{2}} y_{1}(z)\right],
\end{aligned}
$$

satisfies the Fuchsian system $D_{Y}\left(\kappa_{2}+\theta_{0}, \kappa_{2}+\theta_{1}, \kappa_{2}+\theta_{t}, \kappa_{2}+\theta_{\infty} ; \tilde{\lambda}, \tilde{\mu} ; k\right)$, where $\tilde{\lambda}$ and $\tilde{\mu}$ are defined in equation (4.8).

(iii) If $\kappa_{2} \in \mathbb{Z}_{<0}$ and $y_{1}(z)$ is a solution to $D_{y_{1}}\left(\theta_{0}, \theta_{1}, \theta_{t}, \theta_{\infty} ; \lambda, \mu\right)$, then the function

$$
\tilde{y}(z)=\left(\frac{d}{d z}\right)^{-\kappa_{2}} y_{1}(z)
$$

satisfies $D_{y_{1}}\left(\kappa_{2}+\theta_{0}, \kappa_{2}+\theta_{1}, \kappa_{2}+\theta_{t}, \kappa_{2}+\theta_{\infty} ; \tilde{\lambda}, \tilde{\mu}\right)$.

Proof. (i) If $\nu=-1$, then it follows immediately that the function $U={ }^{t}\left(z^{-1} y_{1}(z), z^{-1} y_{2}(z)\right.$, $\left.(z-1)^{-1} y_{1}(z),(z-1)^{-1} y_{2}(z),(z-t)^{-1} y_{1}(z),(z-t)^{-1} y_{2}(z)\right)$ satisfied equation $(6.1)$ for $n=0$. Assume now that the function $U={ }^{t}\left(u_{1}(z), u_{2}(z), u_{3}(z), u_{4}(z), u_{5}(z), u_{6}(z)\right)$ satisfies equation (6.1). Set $V=d U / d z$. Since

$$
\begin{aligned}
& \frac{B_{0}^{(-1-n)}}{z^{2}}+\frac{B_{1}^{(-1-n)}}{(z-1)^{2}}+\frac{B_{t}^{(-1-n)}}{(z-t)^{2}}=\frac{1}{z} \frac{B_{0}^{(-1-n)}}{z}\left(\begin{array}{c}
u_{1}(z) \\
u_{2}(z) \\
0 \\
0 \\
0 \\
0
\end{array}\right)+\frac{1}{z-1} \frac{B_{0}^{(-1-n)}}{z-1}\left(\begin{array}{c}
0 \\
0 \\
u_{3}(z) \\
u_{4}(z) \\
0 \\
0
\end{array}\right) \\
& +\frac{1}{z-t} \frac{B_{0}^{(-1-n)}}{z-t}\left(\begin{array}{c}
0 \\
0 \\
0 \\
0 \\
u_{5}(z) \\
u_{6}(z)
\end{array}\right)=\frac{1}{z}\left(\begin{array}{c}
u_{1}^{\prime}(z) \\
u_{2}^{\prime}(z) \\
0 \\
0 \\
0 \\
0
\end{array}\right)+\frac{1}{z-1}\left(\begin{array}{c}
0 \\
0 \\
u_{3}^{\prime}(z) \\
u_{4}^{\prime}(z) \\
0 \\
0
\end{array}\right)+\frac{1}{z-t}\left(\begin{array}{c}
0 \\
0 \\
0 \\
0 \\
u_{5}^{\prime}(z) \\
u_{6}^{\prime}(z)
\end{array}\right),
\end{aligned}
$$


we have

$$
\begin{aligned}
\frac{d V}{d z} & =\frac{d}{d z}\left\{\left(\frac{B_{0}^{(-1-n)}}{z}+\frac{B_{1}^{(-1-n)}}{z-1}+\frac{B_{t}^{(-1-n)}}{z-t}\right) U\right\} \\
& =-\left(\frac{B_{0}^{(-1-n)}}{z^{2}}+\frac{B_{1}^{(-1-n)}}{(z-1)^{2}}+\frac{B_{t}^{(-1-n)}}{(z-t)^{2}}\right) U+\left(\frac{B_{0}^{(-1-n)}}{z}+\frac{B_{1}^{(-1-n)}}{z-1}+\frac{B_{t}^{(-1-n)}}{z-t}\right) V \\
& =\left(\frac{B_{0}^{(-2-n)}}{z}+\frac{B_{1}^{(-2-n)}}{z-1}+\frac{B_{t}^{(-2-n)}}{z-t}\right) V .
\end{aligned}
$$

Hence $(i)$ is proved inductively.

(ii) Let $A_{0}, A_{1}, A_{t}$ be the matrices defined by equation (2.5) and set $\nu=\kappa_{2}$. We define the matrix $S$ by equation (4.4) and set $\tilde{U}=S^{-1} U$. Then $\tilde{Y}={ }^{t}\left(\tilde{u}_{1}(z), \tilde{u}_{2}(z)\right)$ satisfies the Fuchsian differential equation $D_{Y}\left(\tilde{\theta}_{0}, \tilde{\theta}_{1}, \tilde{\theta}_{t}, \tilde{\theta}_{\infty} ; \tilde{\lambda}, \tilde{\mu} ; \tilde{k}\right)$ where the parameters are determined by equation (4.5), and $\tilde{u}_{1}(z), \tilde{u}_{2}(z)$ are expressed as equation (4.6). By a straightforward calculation as obtaining equation (4.7), we have equation (6.2).

(iii) follows from $(i i)$.

Note that $(i)$ is valid for Fuchsian differential systems of arbitrary size and arbitrary number of regular singularities. We have a similar statement for the case $\nu=\kappa_{1}$ and $\kappa_{1} \in \mathbb{Z}_{<0}$. Namely, if $\kappa_{1} \in \mathbb{Z}_{<0}$ and $Y={ }^{t}\left(y_{1}(z), y_{2}(z)\right)$ is a solution to the Fuchsian differential equation $D_{Y}\left(\theta_{0}, \theta_{1}, \theta_{t}, \theta_{\infty} ; \lambda, \mu ; k\right)$ (see equation $\left.(2.9)\right)$, then the function $\tilde{Y}={ }^{t}\left(\tilde{y}_{1}(z), \tilde{y}_{2}(z)\right)$ defined by

$$
\begin{aligned}
& \tilde{y}_{1}(z)=\left(\frac{d}{d z}\right)^{-\kappa_{1}-1}\left\{\frac{1}{z-\lambda}\left(\kappa_{1} y_{1}(z)+(z-\tilde{\lambda}) \frac{d y_{1}(z)}{d z}\right)\right\}, \\
& \tilde{y}_{2}(z)=\left(\frac{d}{d z}\right)^{-\kappa_{1}} y_{2}(z)
\end{aligned}
$$

satisfies the Fuchsian system $D_{Y}\left(\kappa_{1}+\theta_{0}, \kappa_{1}+\theta_{1}, \kappa_{1}+\theta_{t},-\kappa_{2} ; \lambda+\kappa_{1} / \mu, \mu ; k\right)$.

If $\kappa_{2}=0$ (resp. $\left.\kappa_{1}=0\right)$, then the Fuchsian system $D_{Y}\left(\kappa_{2}+\theta_{0}, \kappa_{2}+\theta_{1}, \kappa_{2}+\theta_{t}, \kappa_{2}+\theta_{\infty} ; \tilde{\lambda}, \tilde{\mu} ; k\right)$ (resp. $D_{Y}\left(\kappa_{1}+\theta_{0}, \kappa_{1}+\theta_{1}, \kappa_{1}+\theta_{t},-\kappa_{2} ; \lambda+\kappa_{1} / \mu, \mu ; k\right)$ ) coincides with $D_{Y}\left(\theta_{0}, \theta_{1}, \theta_{t}, \theta_{\infty} ; \lambda, \mu ; k\right)$, and the function $\tilde{Y}={ }^{t}\left(\tilde{y}_{1}(z), \tilde{y}_{2}(z)\right)$ just corresponds to ${ }^{t}\left(y_{1}(z), y_{2}(z)\right)$.

On the case $\kappa_{2} \in \mathbb{Z}_{>0}$, we have the following proposition:

Proposition 5. Let $p \in\{0,1, t, \infty\}$ and $C_{p}$ be the cycle starting from $w=z$, turning $w=p$ anti-clockwise and return to $w=z$.

(i) If $\kappa_{2} \in \mathbb{Z}_{>0}$ and $Y={ }^{t}\left(y_{1}(z), y_{2}(z)\right)$ is a solution to the Fuchsian system $D_{Y}\left(\theta_{0}, \theta_{1}, \theta_{t}, \theta_{\infty}\right.$; $\lambda, \mu ; k)$, then the function $\tilde{Y}={ }^{t}\left(\tilde{y}_{1}(z), \tilde{y}_{2}(z)\right)$ defined by

$$
\begin{aligned}
& \tilde{y}_{1}(z)=\int_{C_{p}} \frac{d y_{1}(w)}{d w}(z-w)^{\kappa_{2}} d w \\
& \tilde{y}_{2}(z)=\frac{\kappa_{2} \lambda(\lambda-1)(\lambda-t)}{k(\lambda-\tilde{\lambda})} \int_{C_{p}}\left\{\left(\frac{d y_{1}(w)}{d w}-\mu y_{1}(w)\right) \frac{1}{\lambda-w}+\frac{\mu}{\kappa_{1}} \frac{d y_{1}(w)}{d w}\right\}(z-w)^{\kappa_{2}} d w
\end{aligned}
$$

satisfies the Fuchsian system $D_{Y}\left(\kappa_{2}+\theta_{0}, \kappa_{2}+\theta_{1}, \kappa_{2}+\theta_{t}, \kappa_{2}+\theta_{\infty} ; \tilde{\lambda}, \tilde{\mu} ; k\right)$ for $p \in\{0,1, t, \infty\}$ where $\tilde{\lambda}$ and $\tilde{\mu}$ are defined in equation (4.8).

(ii) If $\kappa_{2} \in \mathbb{Z}_{>0}$ and $y_{1}(z)$ is a solution to $D_{y_{1}}\left(\theta_{0}, \theta_{1}, \theta_{t}, \theta_{\infty} ; \lambda, \mu\right)$, then the function

$$
\tilde{y}(z)=\int_{C_{p}} y_{1}(w)(z-w)^{\kappa_{2}-1} d w
$$

satisfies $D_{y_{1}}\left(\kappa_{2}+\theta_{0}, \kappa_{2}+\theta_{1}, \kappa_{2}+\theta_{t}, \kappa_{2}+\theta_{\infty} ; \tilde{\lambda}, \tilde{\mu}\right)$ for $p \in\{0,1, t, \infty\}$. 
Note that the functions $\tilde{y}_{1}(z), \tilde{y}_{2}(z), \tilde{y}(z)$ may not be polynomials although the integrands of equations (6.3) and (6.4) are polynomials in $z$.

Proof. Set

$$
\begin{aligned}
& K_{1}(w)=\frac{d y_{1}(w)}{d w} \\
& K_{2}(w)=\frac{\kappa_{2} \lambda(\lambda-1)(\lambda-t)}{k(\lambda-\tilde{\lambda})}\left\{\left(\frac{d y_{1}(w)}{d w}-\mu y_{1}(w)\right) \frac{1}{\lambda-w}+\frac{\mu}{\kappa_{1}} \frac{d y_{1}(w)}{d w}\right\} .
\end{aligned}
$$

It follows from Theorem 1 that the function $Y(z)=\left(\begin{array}{c}\tilde{y}_{1}(z) \\ \tilde{y}_{2}(z)\end{array}\right)$ defined by

$$
\begin{aligned}
\tilde{y}_{i}(z) & =\int_{\gamma_{z} \gamma_{p} \gamma_{z}^{-1} \gamma_{p}^{-1}} K_{i}(w)(z-w)^{\kappa_{2}} d w \\
& =\left(1-e^{2 \pi \sqrt{-1} \kappa_{2}}\right) \int_{\gamma_{p}} K_{i}(w)(z-w)^{\kappa_{2}} d w+\int_{\gamma_{z}}\left(K_{i}^{\gamma_{p}}(w)-K_{i}(w)\right)(z-w)^{\kappa_{2}} d w \quad(i=1,2)
\end{aligned}
$$

is a solution to $D_{Y}\left(\kappa_{2}+\theta_{0}, \kappa_{2}+\theta_{1}, \kappa_{2}+\theta_{t}, \kappa_{2}+\theta_{\infty} ; \tilde{\lambda}, \tilde{\mu} ; k\right)$ for $p \in\{0,1, t\}$, where $K_{i}^{\gamma_{p}}(w)$ is the function analytically continued along the cycle $\gamma_{p}$.

If $\kappa_{2}>-1$, then the integrals $\int_{\gamma_{z}}\left(K_{i}^{\gamma_{p}}(w)-K_{i}(w)\right)(z-w)^{\kappa_{2}} d w$ tends to zero as the base point $o$ of the integral tends to $z$, and it follows that the function $\left(\begin{array}{c}\int_{C_{p}} K_{1}(w)(z-w)^{\kappa_{2}} d w \\ \int_{C_{p}} K_{2}(w)(z-w)^{\kappa_{2}} d w\end{array}\right)$ is a solution to $D_{Y}\left(\kappa_{2}+\theta_{0}, \kappa_{2}+\theta_{1}, \kappa_{2}+\theta_{t}, \kappa_{2}+\theta_{\infty} ; \tilde{\lambda}, \tilde{\mu} ; k\right)$ for $\kappa_{2} \in \mathbb{R}_{>-1} \backslash \mathbb{Z}_{>-1}$. By considering the limit $\kappa_{2} \rightarrow n, n \in \mathbb{Z}_{>0}$, we obtain (i) for $p \in\{0,1, t\}$. The case $p=\infty$ follows from $C_{\infty}=C_{t}^{-1} C_{1}^{-1} C_{0}^{-1}$.

By integration by parts as equation (4.9) we obtain (ii).

We have similar proposition for the case $\nu=\kappa_{1}$ and $\kappa_{1} \in \mathbb{Z}_{>0}$. On middle convolution $m c_{\nu}$ for Fuchsian differential systems of arbitrary size and arbitrary number of regular singularities whose parameter $\nu$ is positive integer, the contour $\left[\gamma_{z}, \gamma_{p}\right]$ can be replaced by $C_{p}$.

We can reformulate Theorem 3 (resp. Theorem 4 ) for the case $k_{2} \in \mathbb{Z}_{<0}$ (resp. $k_{1} \in \mathbb{Z}_{<0}$ ) by changing the integral to successive differential and for the case $k_{2} \in \mathbb{Z}_{>0}$ (resp. $k_{1} \in \mathbb{Z}_{>0}$ ) by changing the contour $\left[\gamma_{z}, \gamma_{p}\right]$ to $C_{p}$. The corresponding setting for Heun's equation is described as follows:

Proposition 6. Let $v(w)$ be a solution to Heun's equation written as equation (5.2) with the parameters in equation (5.1).

(i) If $\eta \in \mathbb{Z}_{>1}$, then the function

$$
y(z)=(d / d z)^{\eta-1} v(z)
$$

is a solution to Heun's equation (5.3).

(ii) If $\eta \in \mathbb{Z}_{<1}$, then the function

$$
y(z)=\int_{C_{p}} v(w)(z-w)^{-\eta} d w
$$

is a solution to Heun's equation (5.3) for $p \in\{0,1, t, \infty\}$.

The generalized Darboux transformation (Crum-Darboux transformation) for elliptical representation of Heun's equation was introduced in [29], and we can show that Proposition $6(i)$ 
gives another description of the generalized Darboux transformation. Hence the integral transformation given by Theorem 2 can be regarded as a generalization of the generalized Darboux transformation to non-integer cases. Khare and Sukhatme [15] conjectured a duality of quasiexactly solvable (QES) eigenvalues for elliptical representation of Heun's equation. By rewriting parameters of the duality to Heun's equation on the Riemann sphere, we obtain a correspondence on the parameters $\alpha, \beta, \gamma, \delta, \epsilon, q$ and $\alpha^{\prime}, \beta^{\prime}, \gamma^{\prime}, \delta^{\prime}, \epsilon^{\prime}, q^{\prime}$ on the integral transformation of Heun's equation in Theorem 2. We will report further from a viewpoint of monodromy in a separated paper.

\section{A Appendix}

We investigate the realization of the Fuchsian system (equation (2.1)) for the cases $\lambda=0,1, t, \infty$ in the setting of Section 2 and observe relationships with the lines $L_{0}, L_{0}^{*}, L_{1}, L_{1}^{*}, L_{t}, L_{t}^{*}, L_{\infty}$, $L_{\infty}^{*}$ (see equation (3.3)) in the space of initial conditions $E(t)$.

We consider the case $\lambda=0$, i.e., the case $a_{12}^{(0)}=0, a_{12}^{(1)} \neq 0, a_{12}^{(t)} \neq 0,(t+1) a_{12}^{(0)}+t a_{12}^{(1)}+a_{12}^{(t)} \neq 0$ (see equation (2.4)). Since $a_{12}^{(0)}=0$ and the eigenvalues of $A_{0}$ are $\theta_{0}$ and 0 , the matrix $A_{0}$ is written as

$$
A_{0}=\left(\begin{array}{ll}
\theta_{0} & 0 \\
v & 0
\end{array}\right) \quad \text { or } \quad A_{0}=\left(\begin{array}{ll}
0 & 0 \\
v & \theta_{0}
\end{array}\right),
$$

and it follows from $a_{12}^{(1)} \neq 0, a_{12}^{(t)} \neq 0$ that the matrices $A_{1}, A_{t}$ may be expressed as equation (2.5). We determine $w_{1}, w_{t}$ so as to satisfy $a_{12}(z)=-w_{1}(z-1)-w_{t} /(z-t)=k /(z(z-1))$. Then we have

$$
w_{1}=k /(t-1), \quad w_{t}=-k /(t-1) .
$$

On the case

$$
\begin{aligned}
& A_{0}=\left(\begin{array}{ll}
0 & 0 \\
v & \theta_{0}
\end{array}\right), \quad A_{1}=\left(\begin{array}{ll}
u_{1}+\theta_{1} & -w_{1} \\
u_{1}\left(u_{1}+\theta_{1}\right) / w_{1} & -u_{1}
\end{array}\right), \\
& A_{t}=\left(\begin{array}{ll}
u_{t}+\theta_{t} & -w_{t} \\
u_{t}\left(u_{t}+\theta_{t}\right) / w_{t} & -u_{t}
\end{array}\right),
\end{aligned}
$$

we determine $u_{1}, u_{t}$ so as to satisfy equation (2.2), namely

$$
\begin{aligned}
& v+u_{1}\left(u_{1}+\theta_{1}\right) / w_{1}+u_{t}\left(u_{t}+\theta_{t}\right) / w_{t}=0, \\
& u_{1}+\theta_{1}+u_{t}+\theta_{t}=-\kappa_{1}, \quad \theta_{0}-u_{1}-u_{t}=-\kappa_{2} .
\end{aligned}
$$

We have

$$
\begin{aligned}
& u_{1}=-\theta_{1}+\frac{1}{\theta_{\infty}-\theta_{0}}\left(\frac{k v}{t-1}-\kappa_{1}\left(\kappa_{1}+\theta_{t}\right)\right), \\
& u_{t}=-\theta_{t}-\frac{1}{\theta_{\infty}-\theta_{0}}\left(\frac{k v}{t-1}+\kappa_{1}\left(\kappa_{1}+\theta_{1}\right)\right) .
\end{aligned}
$$

Hence we have one-parameter realization of equation (A.2) with the prescribed condition. We discuss relationship with the Fuchsian system on the line $L_{0}$. For this purpose, we recall matrices $A_{0}, A_{1}, A_{t}$ determined by equations (2.5), (2.7), (2.8) and restrict them to $\lambda=0$. Then all elements in $A_{0}, A_{1}$ and $A_{t}$ are well-defined and we have

$$
\left.A_{0}\right|_{\lambda=0}=\left(\begin{array}{ll}
0 & 0 \\
\theta_{0}\left\{t\left(\theta_{0}-\theta_{\infty}\right) \mu+\kappa_{1}\left(\kappa_{1}+\theta_{1}+t\left(\kappa_{1}+\theta_{t}\right)\right)\right\} /\left(k \theta_{\infty}\right) & \theta_{0}
\end{array}\right) .
$$


In fact the matrices $A_{0}, A_{1}, A_{t}$ restricted to the line $L_{0}$ coincide with the ones determined by equations (A.2), (A.1), (A.3) and $v=\theta_{0}\left\{t\left(\theta_{0}-\theta_{\infty}\right) \mu+\kappa_{1}\left(\kappa_{1}+\theta_{1}+t\left(\kappa_{1}+\theta_{t}\right)\right)\right\} /\left(k \theta_{\infty}\right)$. Note that the second-order differential equation for the function $y_{1}$ on the case of the matrices in equations (A.2), (A.1), (A.3) is obtained as equation (3.4) by substituting $\mu=\left(k \theta_{\infty} v-\right.$ $\left.\kappa_{1} \theta_{0}\left(\kappa_{1}+\theta_{1}+t\left(\kappa_{1}+\theta_{t}\right)\right)\right) /\left(t \theta_{0}\left(\theta_{0}-\theta_{\infty}\right)\right)$.

On the case

$$
\begin{aligned}
& A_{0}=\left(\begin{array}{ll}
\theta_{0} & 0 \\
v & 0
\end{array}\right), \quad A_{1}=\left(\begin{array}{ll}
u_{1}+\theta_{1} & -w_{1} \\
u_{1}\left(u_{1}+\theta_{1}\right) / w_{1} & -u_{1}
\end{array}\right), \\
& A_{t}=\left(\begin{array}{ll}
u_{t}+\theta_{t} & -w_{t} \\
u_{t}\left(u_{t}+\theta_{t}\right) / w_{t} & -u_{t}
\end{array}\right),
\end{aligned}
$$

$u_{1}, u_{t}$ are determined as

$$
u_{1}=\frac{1}{\theta_{\infty}+\theta_{0}}\left(\frac{k v}{t-1}-\kappa_{2}\left(\kappa_{2}+\theta_{t}\right)\right), u_{t}=\frac{-1}{\theta_{\infty}+\theta_{0}}\left(\frac{k v}{t-1}+\kappa_{2}\left(\kappa_{2}+\theta_{1}\right)\right),
$$

to satisfy equation (2.2). To realize the Fuchsian system on the line $L_{0}^{*}$, we recall matrices $A_{0}$, $A_{1}, A_{t}$ determined by equations $(2.5),(2.7),(2.8)$, transform $(\lambda, \mu)\left(=\left(q_{0}, p_{0}\right)\right)$ to $\left(q_{1}, p_{1}\right)$ by equation (3.2) and restrict matrix elements to $q_{1}=0$. Then the matrices $A_{0}, A_{1}$ and $A_{t}$ are determined as equations (A.4), (A.1), (A.5), where

$$
v=\left\{-t\left(\theta_{0}+\theta_{\infty}\right) q_{1}+\theta_{0}\left(\kappa_{1}+\theta_{0}\right)\left(\left(\kappa_{2}+\theta_{t}\right)+t\left(\kappa_{2}+\theta_{1}\right)\right)\right\} /\left(k \theta_{\infty}\right) .
$$

Note that the second-order differential equation for the function $\tilde{y}_{1}=z^{-1} y_{1}$ on the case of the matrices in equations (A.4), (A.1), (A.5) is obtained as equation (3.8) by substituting equation (A.6).

We consider the case $\lambda=1$, i.e., the case $a_{12}^{(1)}=0, a_{12}^{(0)} \neq 0, a_{12}^{(t)} \neq 0,(t+1) a_{12}^{(0)}+t a_{12}^{(1)}+a_{12}^{(t)} \neq 0$. Since $a_{12}^{(1)}=0$ and the eigenvalues of $A_{1}$ are $\theta_{1}$ and 0 , the matrix $A_{1}$ is written as

$$
A_{1}=\left(\begin{array}{ll}
\theta_{1} & 0 \\
v & 0
\end{array}\right) \quad \text { or } \quad A_{1}=\left(\begin{array}{ll}
0 & 0 \\
v & \theta_{1}
\end{array}\right)
$$

and the matrices $A_{0}, A_{t}$ may be expressed as equation (2.5). To satisfy $a_{12}(z)=-w_{0} / z-$ $w_{t} /(z-t)=k /(z(z-t))$, we have

$$
w_{0}=k / t, \quad w_{t}=-k / t .
$$

On the case

$$
\begin{aligned}
& A_{1}=\left(\begin{array}{ll}
0 & 0 \\
v & \theta_{1}
\end{array}\right), \quad A_{0}=\left(\begin{array}{ll}
u_{0}+\theta_{0} & -w_{0} \\
u_{0}\left(u_{0}+\theta_{0}\right) / w_{0} & -u_{0}
\end{array}\right), \\
& A_{t}=\left(\begin{array}{ll}
u_{t}+\theta_{t} & -w_{t} \\
u_{t}\left(u_{t}+\theta_{t}\right) / w_{t} & -u_{t}
\end{array}\right),
\end{aligned}
$$

$u_{0}, u_{t}$ are determined as

$$
\begin{aligned}
& u_{0}=-\theta_{0}+\frac{1}{\theta_{\infty}-\theta_{1}}\left(\frac{k v}{t}-\kappa_{1}\left(\kappa_{1}+\theta_{t}\right)\right), \\
& u_{t}=-\theta_{t}-\frac{1}{\theta_{\infty}-\theta_{1}}\left(\frac{k v}{t}+\kappa_{1}\left(\kappa_{1}+\theta_{0}\right)\right),
\end{aligned}
$$


to satisfy equation (2.2). To realize the Fuchsian system on the line $L_{1}$, we recall matrices $A_{0}$, $A_{1}, A_{t}$ determined by equations (2.5), (2.7), (2.8) and restrict matrix elements to $\lambda=1$. Then the matrices $A_{0}, A_{1}$ and $A_{t}$ are determined as equations (A.8), (A.7), (A.9), where

$$
v=\theta_{1}\left\{(1-t)\left(\theta_{1}-\theta_{\infty}\right) \mu-\kappa_{1}\left(\kappa_{1}+\theta_{0}+(1-t)\left(\kappa_{1}+\theta_{t}\right)\right)\right\} /\left(k \theta_{\infty}\right) .
$$

Note that the second-order differential equation for the function $y_{1}$ on the case of the matrices in equations (A.8), (A.7), (A.9) is obtained as equation (3.5) by substituting equation (A.10).

On the case

$$
\begin{aligned}
& A_{1}=\left(\begin{array}{ll}
\theta_{1} & 0 \\
v & 0
\end{array}\right), \quad A_{0}=\left(\begin{array}{ll}
u_{0}+\theta_{0} & -w_{0} \\
u_{0}\left(u_{0}+\theta_{0}\right) / w_{0} & -u_{0}
\end{array}\right), \\
& A_{t}=\left(\begin{array}{ll}
u_{t}+\theta_{t} & -w_{t} \\
u_{t}\left(u_{t}+\theta_{t}\right) / w_{t} & -u_{t}
\end{array}\right),
\end{aligned}
$$

$u_{0}, u_{t}$ are determined as

$$
u_{0}=\frac{1}{\theta_{\infty}+\theta_{1}}\left(\frac{k v}{t}-\kappa_{2}\left(\kappa_{2}+\theta_{t}\right)\right), \quad u_{t}=\frac{-1}{\theta_{\infty}+\theta_{1}}\left(\frac{k v}{t}+\kappa_{2}\left(\kappa_{2}+\theta_{0}\right)\right),
$$

to satisfy equation (2.2). To realize the Fuchsian system on the line $L_{1}^{*}$, we recall matrices $A_{0}$, $A_{1}, A_{t}$ determined by equations (2.5), (2.7), (2.8), transform $(\lambda, \mu)\left(=\left(q_{0}, p_{0}\right)\right)$ to $\left(q_{2}, p_{2}\right)$ by equation (3.2) and restrict matrix elements to $q_{2}=0$. Then the matrices $A_{0}, A_{1}$ and $A_{t}$ are determined as equations (A.11), (A.7), (A.12), where

$$
v=\left\{(t-1)\left(\theta_{1}+\theta_{\infty}\right) q_{2}-\theta_{1}\left(\kappa_{1}+\theta_{1}\right)\left(\left(\kappa_{2}+\theta_{t}\right)+(1-t)\left(\kappa_{2}+\theta_{0}\right)\right)\right\} /\left(k \theta_{\infty}\right) .
$$

Note that the second-order differential equation for the function $\tilde{y}_{1}=(z-1)^{-1} y_{1}$ on the case of the matrices in equations (A.11), (A.7), (A.12) is obtained as equation (3.9) by substituting equation (A.13).

We consider the case $\lambda=t$, i.e., the case $a_{12}^{(t)}=0, a_{12}^{(0)} \neq 0, a_{12}^{(1)} \neq 0,(t+1) a_{12}^{(0)}+t a_{12}^{(1)}+a_{12}^{(t)} \neq 0$. Then the matrix $A_{t}$ is written as

$$
A_{t}=\left(\begin{array}{cc}
\theta_{t} & 0 \\
v & 0
\end{array}\right) \quad \text { or } \quad A_{t}=\left(\begin{array}{cc}
0 & 0 \\
v & \theta_{t}
\end{array}\right)
$$

and the matrices $A_{0}, A_{1}$ may be expressed as equation (2.5). To satisfy $a_{12}(z)=-w_{0} / z-$ $w_{1} /(z-1)=k /(z(z-1))$, we have

$$
w_{0}=k, \quad w_{1}=-k .
$$

On the case

$$
\begin{aligned}
& A_{t}=\left(\begin{array}{ll}
0 & 0 \\
v & \theta_{t}
\end{array}\right), \quad A_{0}=\left(\begin{array}{ll}
u_{0}+\theta_{0} & -w_{0} \\
u_{0}\left(u_{0}+\theta_{0}\right) / w_{0} & -u_{0}
\end{array}\right), \\
& A_{1}=\left(\begin{array}{ll}
u_{1}+\theta_{1} & -w_{1} \\
u_{1}\left(u_{1}+\theta_{1}\right) / w_{1} & -u_{1}
\end{array}\right),
\end{aligned}
$$

$u_{0}, u_{1}$ are determined as

$$
\begin{aligned}
& u_{0}=-\theta_{0}+\frac{1}{\theta_{\infty}-\theta_{t}}\left(k v-\kappa_{1}\left(\kappa_{1}+\theta_{1}\right)\right), \\
& u_{1}=-\theta_{1}-\frac{1}{\theta_{\infty}-\theta_{t}}\left(k v+\kappa_{1}\left(\kappa_{1}+\theta_{0}\right)\right),
\end{aligned}
$$


to satisfy equation (2.2). To realize the Fuchsian system on the line $L_{t}$, we recall matrices $A_{0}$, $A_{1}, A_{t}$ determined by equations (2.5), (2.7), (2.8) and restrict matrix elements to $\lambda=t$. Then the matrices $A_{0}, A_{1}$ and $A_{t}$ are determined as equations (A.15), (A.14), (A.16), where

$$
v=\theta_{t}\left\{t(t-1)\left(\theta_{t}-\theta_{\infty}\right) \mu-\kappa_{1}\left(t\left(\kappa_{1}+\theta_{0}\right)+(t-1)\left(\kappa_{1}+\theta_{1}\right)\right)\right\} /\left(k \theta_{\infty}\right) .
$$

Note that the second-order differential equation for the function $y_{1}$ on the case of the matrices in equations (A.15), (A.14), (A.16) is obtained as equation (3.6) by substituting equation (A.17).

On the case

$$
\begin{aligned}
A_{t} & =\left(\begin{array}{ll}
\theta_{t} & 0 \\
v & 0
\end{array}\right), \quad A_{0}=\left(\begin{array}{ll}
u_{0}+\theta_{0} & -w_{0} \\
u_{0}\left(u_{0}+\theta_{0}\right) / w_{0} & -u_{0}
\end{array}\right), \\
A_{1} & =\left(\begin{array}{ll}
u_{1}+\theta_{1} & -w_{1} \\
u_{1}\left(u_{1}+\theta_{1}\right) / w_{1} & -u_{1}
\end{array}\right),
\end{aligned}
$$

$u_{0}, u_{t}$ are determined as

$$
u_{0}=\frac{1}{\theta_{\infty}+\theta_{t}}\left(k v-\kappa_{2}\left(\kappa_{2}+\theta_{1}\right)\right), \quad u_{1}=\frac{-1}{\theta_{\infty}+\theta_{t}}\left(k v+\kappa_{2}\left(\kappa_{2}+\theta_{0}\right)\right),
$$

to satisfy equation (2.2). To realize the Fuchsian system on the line $L_{t}^{*}$, we recall matrices $A_{0}$, $A_{1}, A_{t}$ determined by equations $(2.5),(2.7),(2.8)$, transform $(\lambda, \mu)\left(=\left(q_{0}, p_{0}\right)\right)$ to $\left(q_{3}, p_{3}\right)$ by equation (3.2) and restrict matrix elements to $q_{3}=0$. Then the matrices $A_{0}, A_{1}$ and $A_{t}$ are determined as equations (A.18), (A.14), (A.19), where

$$
v=\left\{t(1-t)\left(\theta_{t}+\theta_{\infty}\right) q_{3}-\theta_{t}\left(\kappa_{1}+\theta_{t}\right)\left(t\left(\kappa_{2}+\theta_{1}\right)+(t-1)\left(\kappa_{2}+\theta_{0}\right)\right)\right\} /\left(k \theta_{\infty}\right) .
$$

Note that the second-order differential equation for the function $\tilde{y}_{1}=(z-1)^{-1} y_{1}$ on the case of the matrices in equations (A.18), (A.14), (A.19) is obtained as equation (3.10) by substituting equation (A.20).

We consider the case $\lambda=\infty$, i.e., the case $a_{12}^{(0)} \neq 0, a_{12}^{(1)} \neq 0, a_{12}^{(t)} \neq 0,(t+1) a_{12}^{(0)}+t a_{12}^{(1)}+$ $a_{12}^{(t)}=0$. We can set $A_{0}, A_{1}, A_{t}$ as equation (2.5) and we determine $u_{0}, u_{1}, u_{t}, w_{0}, w_{1}, w_{t}$ to satisfy equation $(2.2)$ and

$$
a_{12}(z)=-\frac{w_{0}}{z}-\frac{w_{1}}{z-1}-\frac{w_{t}}{z-t}=\frac{k}{z(z-1)(z-t)}
$$

Then we have

$$
w_{0}=-k / t, \quad w_{1}=k /(t-1), \quad w_{t}=-k /(t(t-1)),
$$

and other relations are written as

$$
\begin{aligned}
& u_{0}\left(u_{0}+\theta_{0}\right) / w_{0}+u_{1}\left(u_{1}+\theta_{1}\right) / w_{1}+u_{t}\left(u_{t}+\theta_{t}\right) / w_{t}=0, \\
& u_{0}+\theta_{0}+u_{1}+\theta_{1}+u_{t}+\theta_{t}=-\kappa_{1}, \quad-u_{0}-u_{1}-u_{t}=-\kappa_{2} .
\end{aligned}
$$

We solve the equation for $u_{0}, u_{1}, u_{t}$ by adding one more relation $-\left(u_{1}+\theta_{1}+t\left(u_{t}+\theta_{t}\right)\right)=l$. We have

$$
\begin{aligned}
& u_{0}=-\theta_{0}-\kappa_{1}+\frac{\tilde{l}}{t \theta_{\infty}}, \quad u_{1}=-\theta_{1}+\frac{\tilde{l}-\theta_{\infty} l}{(1-t) \theta_{\infty}}, \quad u_{t}=-\theta_{t}+\frac{\tilde{l}-t \theta_{\infty} l}{t(t-1) \theta_{\infty}} \\
& \tilde{l}=l^{2}+\left(\theta_{1}+t \theta_{t}\right) l+t \kappa_{1}\left(\kappa_{1}+\theta_{0}\right) .
\end{aligned}
$$


The second-order differential equation for the function $y_{1}$ is written as

$$
\begin{aligned}
& \frac{d^{2} y_{1}}{d z^{2}}+\left(\frac{1-\theta_{0}}{z}+\frac{1-\theta_{1}}{z-1}+\frac{1-\theta_{t}}{z-t}\right) \frac{d y_{1}}{d z}+\frac{\kappa_{1}\left(\kappa_{2}+2\right) z-q}{z(z-1)(z-t)} y_{1}=0, \\
& q=l\left(\theta_{\infty}-1\right)+\kappa_{1}\left(t\left(\kappa_{2}+\theta_{t}+1\right)+\kappa_{2}+\theta_{1}+1\right) .
\end{aligned}
$$

To realize the Fuchsian system on the line $L_{\infty}$, we recall matrices $A_{0}, A_{1}, A_{t}$ determined by equations $(2.5),(2.7),(2.8)$, transform $(\lambda, \mu)\left(=\left(q_{0}, p_{0}\right)\right)$ to $\left(q_{\infty}, p_{\infty}\right)$ by equation (3.2), replace $k$ by $-k q_{\infty}$ and restrict matrix elements to $q_{\infty}=0$. Then the matrices $A_{0}, A_{1}$ and $A_{t}$ are determined as equations (2.5), (A.21), (A.22), where

$$
l=p_{\infty}
$$

We have observed that Fuchsian systems on the lines $L_{0}, L_{0}^{*}, L_{1}, L_{1}^{*}, L_{t}, L_{t}^{*}, L_{\infty}$ are realized by Fuchsian systems for the case $\lambda=0,1, t, \infty$. Here the case $L_{\infty}^{*}$ is missing. In fact this case does not simply correspond with the Fuchsian system as equation (2.1), because we have

$$
\begin{aligned}
& u_{0}=\frac{1}{t \theta_{\infty}} \frac{1}{p_{4}^{2}}+O\left(p_{4}^{-1}\right), \quad u_{1}=\frac{1}{(1-t) \theta_{\infty}} \frac{1}{p_{4}^{2}}+O\left(p_{4}^{-1}\right), \\
& u_{t}=\frac{1}{t(t-1) \theta_{\infty}} \frac{1}{p_{4}^{2}}+O\left(p_{4}^{-1}\right),
\end{aligned}
$$

as $p_{4} \rightarrow 0$ in the coordinate $\left(q_{4}, p_{4}\right)$ in equation $(3.2)$, although we can restrict the second-order differential equation for $y_{1}$ to $p_{4}=0$ as equation (3.11).

\section{Acknowledgements}

The author would like to thank Alexander Kazakov for sending the paper [14] with valuable comments. The author is supported by the Grant-in-Aid for Young Scientists (B) (No. 19740089) from the Japan Society for the Promotion of Science.

\section{References}

[1] Boalch P., From Klein to Painlevé via Fourier, Laplace and Jimbo, Proc. London Math. Soc. (3) 90 (2005), 167-208, math.AG/0308221.

[2] Dettweiler M., Reiter S., An algorithm of Katz and its application to the inverse Galois problem. Algorithmic methods in Galois theory, J. Symbolic Comput. 30 (2000), 761-798.

[3] Dettweiler M., Reiter S., Middle convolution of Fuchsian systems and the construction of rigid differential systems, J. Algebra 318 (2007), 1-24.

[4] Dettweiler M., Reiter S., Painlevé equations and the middle convolution, Adv. Geom. 7 (2007), 317-330, math.AG/0605384.

[5] Filipuk G., On the middle convolution and birational symmetries of the sixth Painlevé equation, Kumamoto J. Math. 19 (2006), 15-23.

[6] Gesztesy F., Weikard R., Treibich-Verdier potentials and the stationary (m)KdV hierarchy, Math. Z. 219 (1995), 451-476.

[7] Haraoka Y., Filipuk G., Middle convolution and deformation for Fuchsian systems, J. Lond. Math. Soc. (2) 76 (2007), 438-450.

[8] Inaba M., Iwasaki K., Saito, M., Moduli of stable parabolic connections, Riemann-Hilbert correspondence and geometry of Painlevé equation of type VI. I, Publ. Res. Inst. Math. Sci. 42 (2006), 987-1089, math.AG/0309342.

[9] Inaba M., Iwasaki K., Saito, M., Moduli of stable parabolic connections, Riemann-Hilbert correspondence and geometry of Painlevé equation of type VI. II, in Moduli Spaces and Arithmetic Geometry, Adv. Stud. Pure Math., Vol. 45, Math. Soc. Japan, Tokyo, 2006, 387-432, math.AG/0605025. 
[10] Iwasaki K., Kimura H., Shimomura S., Yoshida M., From Gauss to Painlevé. A modern theory of special functions, Friedr. Vieweg \& Sohn, Braunschweig, 1991.

[11] Jimbo M., Miwa T., Monodromy preserving deformation of linear ordinary differential equations with rational coefficients. II, Phys. D 2 (1981), 407-448.

[12] Katz N.M., Rigid local systems, Annals of Mathematics Studies, Vol. 139. Princeton University Press, Princeton, NJ, 1996.

[13] Kazakov A.Ya., Slavyanov S.Yu., Integral relations for special functions of the Heun class, Theoret. and Math. Phys. 107 (1996), 733-739.

[14] Kazakov A.Ya., Slavyanov S.Yu., Euler integral symmetries for a deformed Heun equation and symmetries of the Painlevé PVI equation, Theoret. and Math. Phys. 155 (2008), 721-732.

[15] Khare A., Sukhatme U., Periodic potentials with a finite number of band gaps, J. Math. Phys. 47 (2006), 062103, 22 pages, quant-ph/0602105.

[16] Kitaev A., Special functions of the isomonodromy type. Acta Appl. Math. 64 (2000), 1-32.

[17] Novikov D.P., Integral transformation of solutions of a Fuchs-class equation that corresponds to the Okamoto transformation of the Painlevé VI equation, Theoret. and Math. Phys. 146 (2006), 295-303.

[18] Okamoto K., Sur les feuilletages associés aux équations du second ordre à points critiques fixes de P. Painlevé, Japan. J. Math. (N.S.) 5 (1979), 1-79.

[19] Okamoto K., Studies on the Painlevé equations. I. Sixth Painlevé equation PVI $_{\mathrm{VI}}$ Ann. Mat. Pura Appl. (4) 146 (1987), 337-381.

[20] Okamoto K., Isomonodromic deformation and Painlevé equations, and the Garnier system. J. Fac. Sci. Univ. Tokyo Sect. IA Math. 33 (1986), 575-618.

[21] Ronveaux A. (Editor), Heun's differential equations, Oxford University Press, Oxford, 1995.

[22] Shioda T., Takano K., On some Hamiltonian structures of Painlevé systems. I, Funkcial. Ekvac. 40 (1997), 271-291.

[23] Slavyanov S., Lay W., Special functions. A unified theory based on singularities, Oxford University Press, Oxford, 2000.

[24] Smirnov A.O., Elliptic solitons and Heun's equation, in The Kowalevski property (Leeds, 2000), CRM Proc. Lecture Notes, Vol. 32, Amer. Math. Soc., Providence, RI, 2002, 287-305, math.CA/0109149.

[25] Takemura K., The Heun equation and the Calogero-Moser-Sutherland system. I. The Bethe ansatz method, Comm. Math. Phys. 235 (2003), 467-494, math.CA/0103077.

[26] Takemura K., The Heun equation and the Calogero-Moser-Sutherland system. II. The perturbation and the algebraic solution, Electron. J. Differential Equations 2004 (2004), no. 15, 30 pages, math.CA/0112179.

[27] Takemura K., The Heun equation and the Calogero-Moser-Sutherland system. III. The finite gap property and the monodromy, J. Nonlinear Math. Phys. 11 (2004), 21-46, math.CA/0201208.

[28] Takemura K., The Heun equation and the Calogero-Moser-Sutherland system. IV. The Hermite-Krichever ansatz, Comm. Math. Phys. 258 (2005), 367-403, math.CA/0406141.

[29] Takemura K., The Heun equation and the Calogero-Moser-Sutherland system. V. Generalized Darboux transformations, J. Nonlinear Math. Phys. 13 (2006), 584-611, math.CA/0508093.

[30] Takemura K., Integral representation of solutions to Fuchsian system and Heun's equation, J. Math. Anal. Appl. 342 (2008), 52-69, arXiv:0705.3358.

[31] Treibich A., Verdier J.-L., Revêtements exceptionnels et sommes de 4 nombres triangulaires, Duke Math. J. 68 (1992), 217-236.

[32] Vidunas R., Kitaev A., Schlesinger transformations for algebraic Painlevé VI solutions, arXiv:0810.2766.

[33] Watanabe H., Birational canonical transformations and classical solutions of the sixth Painlevé equation, Ann. Scuola Norm. Sup. Pisa Cl. Sci. (4) 27 (1998), 379-425. 